\title{
Optimal Mechanisms for Selling Information
}

\author{
Moshe Babaioff \\ Microsoft Research SVC \\ moshe@microsoft.com
}

\author{
Robert Kleinberg \\ Cornell University \\ rdk@cs. cornell.edu
}

\author{
Renato Paes Leme* \\ Cornell University \\ renatoppl@cs. cornell.edu
}

April 26, 2012

\begin{abstract}
The buying and selling of information is taking place at a scale unprecedented in the history of commerce, thanks to the formation of online marketplaces for user data. Data providing agencies sell user information to advertisers to allow them to match ads to viewers more effectively. In this paper we study the design of optimal mechanisms for a monopolistic data provider to sell information to a buyer, in a model where both parties have (possibly correlated) private signals about a state of the world, and the buyer uses information learned from the seller, along with his own signal, to choose an action (e.g., displaying an ad) whose payoff depends on the state of the world.

We provide sufficient conditions under which there is a simple one-round protocol (i.e. a protocol where the buyer and seller each sends a single message, and there is a single money transfer) achieving optimal revenue. In these cases we present a polynomial-time algorithm that computes the optimal mechanism. Intriguingly, we show that multiple rounds of partial information disclosure (interleaved by payment to the seller) are sometimes necessary to achieve optimal revenue if the buyer is allowed to abort his interaction with the seller prematurely. We also prove some negative results about the inability of simple mechanisms for selling information to approximate more complicated ones in the worst case.
\end{abstract}

\footnotetext{
${ }^{*}$ This work was done while the author was an intern in Microsoft Research SVC. He is supported by a Microsoft Research Fellowship.
} 


\section{Introduction}

A growing trend in online advertisement is the usage of behavioral targeting and user information (like demographics) to better match advertisements to the viewer. This is possible due to the existence of data providing agencies, like Bluekai, Bluecava, eXelate Media, Clearsprings and RapLeaf, whose business consists in collecting, curating and selling information about user intent to advertisers. An article in NYT [8] analyzes this phenomenon and points out that data agencies are not exclusively an Internet phenomenon. For example, for many years companies like Acxiom and Experian (founded in 1969 and 1980 respectively) have been collecting information about consumer habits and selling this information to marketers, who then can use it to send catalogs by mail.

A concrete situation is as follows: an advertiser has multiple different ads that he can present to the viewer, and the effectiveness of each of them depends on both the ad and the characteristics of the viewer. For example, a car maker would rather show sport car advertisement to affluent young bachelors while showing ads for family cars to older viewers with kids. A data providing agency (the seller), might have some information about the viewer generating the impression, like gender, age and past interaction on that site. Such information could be valuable to the advertiser as he would be able to use it for better targeting, and the monopolist seller would like to extract as much as possible out of this value as revenue. The advertiser (buyer) might have some information about the viewer as well, and this information might possibly be correlated with the seller's information. The seller has uncertainty about the buyer's information or utilities, yet possesses some belief about those.

While selling information about viewers raises obvious privacy questions, it also raises fascinating questions of a purely economic nature. How does one quantify the value of this information? What is the optimal (i.e. revenue-maximizing) selling strategy for information? What are the qualitative differences between selling information and selling physical goods and services? How do these differences influence the design of markets for information, and the algorithmic problems underlying such markets?

To highlight the issues inherent in such questions, it is helpful to highlight some differences between a seller offering $n$ distinct goods for sale and a seller offering $n$ bits of information.

(1) A seller of goods can group them into bundles, offering a subset of the goods at a specified price. A seller of bits can do many other things: for example, she 1 can set a specified price for revealing the Boolean XOR of the first two bits or some more complex function of the bits.

(2) A consumer of goods generally knows their value even before they are allocated. The value of a piece of information is typically not known until the information is revealed.

(3) By the taxation principle, a buyer of goods can be assumed, without loss of generality, to be facing a posted-price for each bundle (that is independent of his type). A seller of information may, in some cases, be able to extract strictly more revenue using an interactive protoco 2 rather than posted pricing. (See Section 5.)

To be sure, there are some cases in which trading goods possesses some of the characteristics of trading information noted above. For example, a customer in a restaurant does not necessarily know the quality of the food he is about to consume; in turn, this can lead to sellers using interactive protocols, for example, allowing the restaurant customer to try a limited sample of food for a reduced price (or even for free) before deciding whether to order more. We interpret such situations as markets in which information and goods are coupled together, i.e. revealing the quality of the food occurs in conjunction with selling the food itself.

\footnotetext{
${ }^{1}$ Throughout this paper, we use female pronouns for the seller and male pronouns for the buyer.

${ }^{2}$ In this paper we will use the terms protocol and mechanism interchangeably.
} 
This paper addresses some of these questions raised above, by situating them in a model that eliminates extraneous features - such as coupling of goods and information, or competition between multiple buyers and sellers of information - while attempting to remain quite general in the model's assumptions about information and its utility. Our only such assumption is that the utility of information lies in guiding future actions of the party receiving the information. Thus, in our model there is a single seller and single buyer. A state of the world (denoted by $\omega$ henceforth) is known to the seller but not the buyer 3 , and the buyer's payoff type (denoted by $\theta$ ) is known to the buyer but not the seller. The two parties engage in an interactive protocol, consisting of one or more rounds in which signals and/or money are exchanged. After this interaction, the buyer chooses an action (based on his posterior beliefs) and receives a payoff that depends on the state of the world, his own payoff type, and the chosen action.

Crucially, we assume that the seller designs the protocol and can be trusted to faithfully follow the protocol he designs. The buyer, on the other hand, need not be honest: he may send signals that are inconsistent with his true payoff type if it is rational to do so. We do, however, distinguish between committed buyers - who can be committed to complete the specified protocol even if they are sending dishonest signals - and uncommitted buyers, who may abort the protocol if it is rational to do so, for example when they have received information and not yet paid for it.

Our results. The set of all interactive protocols is a large and ill-structured space. Searching for the revenue-maximizing one is unfathomably complex unless there is a way to limit the search space. Our first set of results provide the tools necessary for that. In mechanism design this is often done by invoking some form of the revelation principle, due to Gibbard [13] and Myerson [16. In their setting, buyers have private types and the seller (mechanism designer) needs to choose among a set of outcomes and can charge payments from the buyers. The revelation principle states that if a certain outcome and payments can be implemented in equilibrium of a possibly complicated and interactive mechanism, then it can be implemented in a simple direct revelation mechanism, where buyers report their types, and the mechanism chooses an outcome and payments. Moreover, this mechanism has a simple equilibrium where each buyer reports his type truthfully. If the outcome is the allocation of traditional goods, the revelation principle implies that the mechanism can be implemented as a protocol consisting of three steps: (i) buyers report their type, (ii) payments take place, (iii) outcome is determined. We say that such a mechanism has the one-round revelation property, a property which we define precisely later (in Definition 2.7), but intuitively it means that the buyers move only once (by declaring their type), payments happen only once and the sellers move only once (by choosing the outcome).

Now, consider the case where instead of an allocation of traditional goods the outcome is the disclosure of information. Myerson's revelation principle still holds in the sense that any outcome can be implemented by a mechanism where buyers truthfully report their type in the first step. It is not clear, however, if the stronger property of one-round revelation still holds. After the buyer report his type, a sequence of payments and partial information disclosures might be required in order to implement a certain outcome.

Our first set of results (Theorems 3.1 and 4.1) provide conditions under which the one-round revelation property holds. Their precise theorem statement, to be given in Sections 3 and 4 , are a bit stronger: they supplement the revelation principle with additional information about the relative timing of signals and payments.

Theorem 1.1 When buyers are committed, or when buyers are uncommitted but $\omega$ and $\theta$ are

\footnotetext{
${ }^{3}$ Our model also incorporates cases in which the buyer and seller each receive (possibly different) signals about the state of the world. In such cases, we simply treat the buyer's signal as being encoded in her payoff type, $\theta$.
} 
independent random variables, any mechanism can be transformed into a mechanism that extracts the same revenue and has the following form: the buyer and seller each sends a single message, payment takes place only once, the buyer's message is simply an announcement of his type, and truthful reporting maximizes the buyer's utility.

Interestingly, the revelation principle fails in the remaining case, when there are uncommitted buyers and correlated signals. The usual logic justifying the revelation principle - that the agents can always report their types to the mechanism and let it simulate their optimal strategy given their type - does not apply for a subtle reason having to do with the timing of payments, the correlation of the signals, and the fact that the buyer is uncommitted. The direct mechanism that attempts to simulate an interactive protocol cannot determine an unbiased estimate of the buyer's expected payment before observing $\omega$, because, unlike in the independent case, the conditional distribution of $\omega$ depends on the value of $\theta$ (the buyer's true type) and not necessarily on the type that is reported. On the other hand, if the mechanism simulates the protocol using the realization of $\omega$ and posts a price that depends on the simulation outcome, this fails because the buyers are uncommitted: the price reveals information about $\omega$, and the buyers may take this information for free while refusing to pay.

Our next results concern algorithms for computing the optimal mechanism. Even when the one-round revelation property holds, it is far from obvious how to compute the optimal mechanism efficiently. In a one-round revelation mechanism, the seller allocates information to the buyer by revealing a (possibly random) signal sampled from a distribution that depends on $\omega$ and $\theta$. The main difficulty is that seller is free to choose the support size of this distribution (i.e., the number of potential signals) and in principle, this leads to an optimization problem of unbounded dimensionality. Nevertheless, we show that the optimal mechanism can be computed in polynomial time by solving a convex program of bounded dimensionality; a by-product of the proof is an explicit upper bound on the number of potential signals.

Theorem 1.2 Suppose that $\omega$ can take only $m$ possible values and $\theta$ can take only $n$ possible values. When buyers are committed, or when buyers are uncommitted but $\omega$ and $\theta$ are independent random variables, there is an algorithm that computes the optimal mechanism in time poly $(m, n)$. Furthermore, there is an optimal mechanism in which every signal transmitted from the seller to a buyer is sampled from a set of size $O(m+n)$.

In Theorem 4.2, we prove an analogue of the result of Cremer and McLean [10] on optimal auctions with correlated bids. We show that when the correlation of $\omega$ and $\theta$ is complex enough that a certain matrix has full rank, the optimal mechanism extracts the full surplus. However, as in [10], when this matrix is ill-conditioned the optimal mechanism can be quite exotic, using a mixture of unboundedly large positive and negative payments. This raises the following question: To what extent can its revenue be approximated by simpler and more natural mechanisms? We explore this question in Section 4.2 by investigating the relative power of four progressively more general types of mechanisms:

(i) a "sealed envelope" mechanism that treats $\omega$ as an indivisible good by writing its value inside a sealed envelope and posts a price for the the envelope;

(ii) mechanisms that reveal a signal about $\omega$ but charge the buyer for this signal before revealing it;

(iii) mechanisms that reveal a signal about $\omega$ and then charge the buyer a non-negative amount that depends on the signal;

(iv) arbitrary mechanisms. 
It is not hard to show that if one compares the optimal mechanisms from two of these four classes, their revenue never differs by more a factor of more than $|\Theta|$, the number of potential buyer types. Section 4.2 shows that this multiplicative gap is tight up to a constant factor: for any two of the aforementioned classes of mechanisms, one can find examples where mechanisms in the more general class obtain $\Omega(|\Theta|)$ times as much revenue as the optimal mechanism in the more specific class.

Our work leaves many interesting open problems, we discuss these problems in Section 6 and point out potential connections to other areas in computer science (as cryptographic) and in economics (as cheap talk and dynamic mechanism design).

Related work. The concept of information occupies a notable position in Auction Theory. In the classic work of Milgrom and Weber [15], the authors consider different auction formats for a single item and discuss how revenue changes as the seller reveals information (but not directly charging for it) regarding the quality of the good. Persico [18] remarks that the information structure is almost always assumed to be exogenous and out of the control of the mechanism designer. He initiates a line of inquire that proposed to endogenize the information acquisition process in the auction. The information acquisition in his model occurs by a competing firm paying some fixed exogenous cost (say by performing R\&D).

It is interesting to consider the qualitative changes when information moves from an auxiliary device to the position of the central object being sold. It was noted by many authors that classic results in economics that were designed for dealing with traditional goods fail when selling information. Varian [21] presents such discussion in a much broader context. Although expressing some similar concerns on the relation with traditional goods, his definition of information is very different from ours.

Closer to our model are the work of Esö and Szentes [12] and Hörner and Skrzypacz [14]. In Esö and Szentes [12] the authors develop a model of consulting, in which the consultant, sells information about a certain random variable to a client. The assumptions on the nature on the random variables are orthogonal to ours: theirs are continuous random variables on $[0,1]$ while ours are discrete random variables. Also, while our utility function is generic, their utility function is linear. The mechanism obtained resembles our Pricing Outcomes mechanism, where the payment of the client depends on the action he takes (in their paper, whether the client decided to undertake a project or not). Given that their utility function is simpler, the structure of the disclosed information is also a lot simpler.

The paper of Hörner and Skrzypacz [14] is close to our model of uncommitted buyers. Their main goal is to design self-enforcing contracts for an agent to sell a binary High-Low signal about the state of the world to a firm. The authors remark: "Lack of commitment creates a hold-up problem: since the Agent is selling information:, once the Firm learns it, it has no reason to pay for it". The model again is orthogonal to ours, since the type of the Firm (information buyer) is known - so there is no uncertainty from the sellers perspective. On the other hand, no side has the ability to commit. This ability in our model leads us to a mechanism design approach to the problem, while the authors analyze the set of equilibria of a game very much in the spirit of the cheap talk literature [5].

Our model is also related the treatment of information in Athey and Levin [3]. The authors model a decision maker who chooses an action and gets a reward depending on both the action chosen and an unknown state of the world from which the decision maker gets an imperfect signal. They assume a total ordering on the space of states of the world and on the space of signals and restrict their attention to monotone decision processes. We, on the other hand, do not make any structural assumptions about the set of possible states of the world and set of possible signals (except being finite) and also do not assume anything about the decision problem. Our approach 
is also different: while the authors in [3] derive comparative statics for the demand of information, we take a mechanism design approach.

Also related to our paper is the work of Admati and Pfleiderer 1, 2. Motivated by financial advice in the market for securities, the authors analyze the following setting: a monopolist seller has information about how much a share of a risky asset pays off, which is distributed according to a normal random variable. They consider a two step process: in the first round, the seller is able to sell this information to a continuum of traders using some mechanism. In the second round, the buyers (traders) trade in a speculative market. The authors consider the problem of how to design mechanisms to maximize revenue. Although of a similar spirit, the model of Admati and Pfleiderer is orthogonal to ours. Their model considers multiple buyers that trade in the same market in the second round, instead of simply making a decision. On the other hand, many features of our model are absent in theirs: they do not consider uncertainty of the seller about the buyer's utility function and the fact that the buyers might already have some private information correlated with the seller's information. Moreover, they also limit the seller's power to transform the signal. In their model, the only transformation the seller is able to perform on the signal is to add normally distributed noise to it.

Our notion of uncommitted buyers is in the spirit of participation constraints used in the literature on dynamic mechanism design [7, 4]. This literature considers multi-round interaction between a mechanism and the agents, and for such mechanisms it is required that individual participation constraints hold in every period. In parallel, when considering uncommitted buyers we require that participation is voluntary at every point the buyer plays, that is, that the buyer does not defect throughout the protocol. Note that unlike that literature, our buyer only gets one exogenous signal, so the different "periods" are not points of getting new exogenous information, but rather points where he takes an action in a multi-round protocol with the seller.

\section{Setting: Context and Protocols}

We consider a setting with a buyer and a monopolist seller. The buyer is a decision maker and his decision can be represented as picking an action $a \in A$. His reward from this action depends on the state of the world which is unobservable. However, both buyer and seller get private signals about the state of the world. Let $\theta \in \Theta$ be the private signal of the buyer and $\omega \in \Omega$ be the private signal of the seller. In this paper we consider the case that both $\Theta$ and $\Omega$ are finite. The buyer's expected reward for taking action $a$ when the two signals are realized to $\theta, \omega$ is given by $u(\theta, \omega, a)$. The pair of private signals comes from a joint distribution $\mu \in \Delta(\Omega \times \Theta)$ U We denote the prior on $\omega$ by the vector $p \in \mathbb{R}_{+}^{\Omega}$, i.e., $p(\omega)=\mu(\omega)$. We call the pair $(u, \mu)$ the context and assume it is common knowledge.

To illustrate the model, suppose the buyer is an Internet advertiser who has acquired one display-ad slot and is deciding which ad to show to the user. So, the set $A$ represents the possible ads he can place on this slot. The effectiveness of each ad depends on who the user is exactly. This is unknown to the buyer, but he has a private signal $\theta$, which is the user browsing history in the website. Consider now the seller as a data provider who has information about age, gender, geographic location and income range of the user. Let this information be encapsulated in a signal $\omega$.

Another interpretation is to consider $\theta$ as the type of the buyer. Since the reward of the buyer $u(\theta, \omega, a)$ is a function of $\theta$, one can use the same model to express the seller's uncertainty about

\footnotetext{
${ }^{4}$ As usual, given any set $X$ we represent the set of probability distributions over $X$ by $\Delta(X)$. We denote by $\mu(\omega, \theta)$ the probability of the event $(\omega, \theta)$ and by $\mu(\omega)=\sum_{\theta} \mu(\omega, \theta)$ and $\mu(\theta)=\sum_{\omega} \mu(\omega, \theta)$ the marginals of $\omega$ and $\theta$ respectively.
} 
the buyer's reward function.

The information that the seller holds is valued by the buyer. If the buyer observe his signal $\theta$ and nothing more, his expected reward is $\max _{a} \mathbb{E}_{\omega}[u(\theta, \omega, a) \mid \theta]$, where the expectation is taken over $\omega$ sampled from $\mu(\cdot \mid \theta)$. If he also learns the value of $\omega$ exactly, his expected reward increases to $\mathbb{E}_{\omega}\left[\max _{a} u(\theta, \omega, a) \mid \theta\right]$. His surplus from knowing $\omega$ is thus:

$$
\xi(\theta)=\mathbb{E}_{\omega}\left[\max _{a} u(\theta, \omega, a) \mid \theta\right]-\max _{a} \mathbb{E}_{\omega}[u(\theta, \omega, a) \mid \theta]
$$

Ideally, the seller would like to extract this extra surplus as revenue, but as she faces uncertainty regarding the buyer (she does not know $\theta$ ) and since the buyer act strategically, generically the seller would not be able to extract all that surplus. The central question of this paper is, "What mechanisms can the seller use in order to extract the largest possible fraction of this surplus?"

\subsection{Sealed Envelope Mechanism}

Before we start exploring the space of all possible mechanisms, we present a very simple (and usually suboptimal) mechanism - the Sealed Envelope Mechanism. We do so in order to highlight some basic difficulties in designing mechanisms for selling information. In this mechanism the seller treats the information as if it were a regular good. She writes $\omega$ on a piece of paper, puts it inside an envelope and then offers the envelope for a fixed price $t$ to the buyer. If the buyer's type is $\theta$, then his value for the envelope equals to $\xi(\theta)$, his surplus from knowing $\omega$, so the revenue is $t \cdot \mathbb{P}_{\theta \sim \mu}[\xi(\theta) \geq t]$. Note that the seller is not using her knowledge of $\omega$ in determining $t$. It is easy to optimize $t$ to obtain the best Sealed Envelope Mechanism.

Notice that, after seeing $\omega$, the seller can update her belief about $\theta$. The seller might try to change the mechanism in the following way: enclose $\omega$ in an envelope and sell it for price $t(\omega)$ maximizing $t \cdot \mathbb{P}_{\theta \sim \mu}[\xi(\theta) \geq t \mid \omega]$. By doing so, the seller leaks information in the prices. Upon observing price $t(\omega)$, the buyer gains information about $\omega$ even without buying the envelope.

\subsection{Generic Interactive Protocol}

Our first step is to define a generic interactive protocol between the buyer and the seller. We assume that the protocol is designed by the seller with only knowledge of the context $(u, \mu)$. The protocol prescribes the behavior of the seller for each $\omega$ and we assume that the seller always follows this prescription. All of this is known to the buyer.

After this we state Myerson's revelation principle for this setting and then we formally define the stronger notion of one-round revelation. Then we discuss to what extend it is possible to obtain one-round revelation mechanisms for this setting.

Definition 2.1 (Generic interactive protocol) A generic interactive protocol for a context $(u, \mu)$ is a finite decision tree defined on a set of nodes $N$. For each non-leaf node, let $C(n)$ be the children of node n. Each non-leaf node is labeled either as a seller-node, as buyer-node or a transfer-node. Furthermore:

- each seller node $n$ has a prescription of the seller behavior, which associates for each $\omega$ a probability distribution over $C(n)$. Formally, the prescription on node $n$ is a collection of distributions $\psi_{n}^{\omega} \in \Delta(C(n))$, one for each $\omega$.

- each transfer node $n$ has only one child and has associated with it a fixed (possibly negative) amount $t(n)$. 
In practice we can think of each edge as labeled with a different message. Starting from the root, if the seller or buyer moves, she or he sends a message, which is represented by moving down the tree (picking a child). As the seller pledges to follow the protocol, her behavior (distribution of messages she sends conditional on $\omega$ ) is encoded in seller nodes in advance. On the other hand, the buyer strategically decides on the messages he sends at buyer nodes given the protocol tree defined by the seller. Moving down the tree from a transfer node $n$ to its child represents a money transfer; the value $t(n)$ designates how much is transferred from buyer to seller, so a negative value represents a payment to the buyer. No action is taken at leaf nodes. In each such a node the buyer will update his belief about $\omega$ based on the protocol history. This belief is called the posterior probability distribution.

We consider two types of strategies for the buyer: committed and uncommitted strategies. A committed strategy is one where we can trust the buyer to follow the entire protocol, i.e., when reaching a buyer node, he sends one of the messages specified by the protocol and when reaching a transfer node, he sends or receives the specified amount of money. An uncommitted strategy is one where the buyer has, on top of that, the option of defecting from the protocol in each node, by simply leaving the mechanism (which is formally captured by allowing him to play " $\perp$ "). More formally:

Definition 2.2 (Buyer strategies) A committed strategy is a collection of distributions $\phi_{n}^{\theta} \in$ $\Delta(C(n))$ for each buyer-node or transfer-node $n$ and each type $\theta$.

An uncommitted strategy is a collection of distributions $\phi_{n}^{\theta} \in \Delta(C(n) \cup\{\perp\})$ for each buyernode or transfer-node $n$ and each type $\theta$.

At this point, it is instructive to represent the Sealed Envelope Mechanism in the form of a generic protocol. It is a tree consisting of three interior nodes: in the root there is a buyer-node with two children corresponding to the messages: "Do not accept the offer" and "Accept the offer". The child corresponding to "Do not accept the offer" is a leaf. The other child is a transfer-node with the specified amount of $t$. Its only child is a seller-node. This seller node has a child for each $\omega \in \Omega$ and the seller prescription for this node is simply $\psi_{n}^{\omega}(\omega)=1$. See Figure 1 for an illustration.

Two other natural selling strategies are important in this work. Pricing Mappings refers to any posted-price mechanism in which the seller presents a menu of offers each having the following form: for a fixed amount of money, the buyer obtains the right to observe a random signal sampled by the seller from a distribution that depends on the value of $\omega$ in a pre-specified way. Pricing Outcomes refers to a similar type of posted-price mechanism with one crucial difference: rather than charging the buyer a fixed amount of money after he selects an offer from the menu, the amount that the buyer pays (or receives) is allowed to depend on the signal that is revealed by the seller. This gives the seller the potential to price-discriminate among buyers whose different types lead to their having different beliefs about the conditional distribution of $\omega$, and therefore different assessments about the expected cost of accepting a given offer. Mechanisms that price mappings or price outputs can easily be represented in the form of generic interactive protocols, as illustrated in Figure 1

It is important to notice that the seller designs the protocol solely based on the context, and after she designs the protocol its description becomes common knowledge. This happens before the pair $(\theta, \omega)$ is drawn. For example, the price at which the item is offered in the Sealed Envelope Mechanism is hard-coded in the protocol, so there is no need for the seller to send a message announcing it.

Now we define the utility associated with a committed strategy for a given protocol. Each committed strategy $\phi$ induces a distribution over the leaves of the tree: sample $(\theta, \omega) \sim \mu$, then start in the root and use $\psi_{n}^{\omega}$ and $\phi_{n}^{\theta}$ to move down the tree until a leaf is reached. Let $Z$ be the leaf 


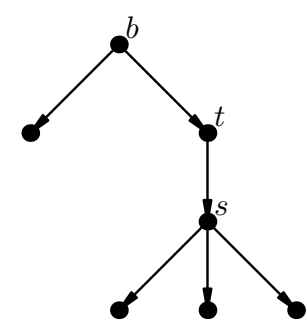

(1)-Sealed-Envelope

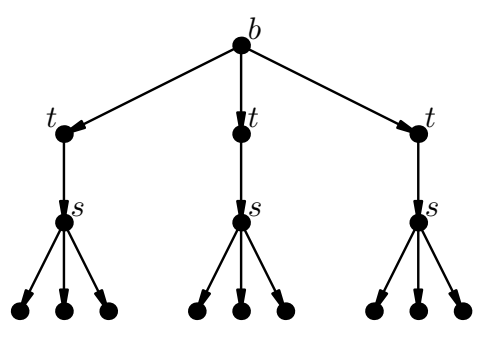

(2)-Pricing-Contracts

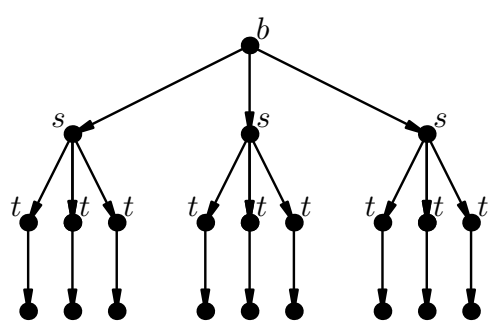

(3)-Pricing-Signals

Figure 1: Illustration of various protocols. A node labeled by $b$ is a buyer node, a node labeled by $s$ is a seller node, and a node labeled by $t$ is a transfer node.

reached. For each leaf $\ell$ associate $\tau(\ell)$ the sum of the amounts of the transfer-nodes in the path between $\ell$ and the root. We define the utility of buyer of type $\theta$ for $\phi$ as:

$$
U(\theta, \phi)=\mathbb{E}_{\omega}\left[\max _{a} \mathbb{E}[u(\theta, \omega, a) \mid \theta, Z]-\tau(Z) \mid \theta\right]
$$

We say that a protocol is voluntary is there is a committed strategy $\phi$ such that

$$
U(\theta, \phi) \geq \max _{a} \mathbb{E}_{\omega}[u(\theta, \omega, a) \mid \theta], \quad \forall \theta .
$$

This means that the expected utility the buyer gets from participating in the protocol is at least as large as the utility he would get by not participating in it. A committed strategy is called optimal if for all $\theta$ and for all alternative committed strategies $\phi^{\prime}, U(\theta, \phi) \geq U\left(\theta, \phi^{\prime}\right)$. The revenue extracted by this protocol is: $\mathbb{E}[\tau(Z)]$.

We can define similar concepts for uncommitted strategies: for a given node $n$, let $\tau(n)$ be the sum of the amounts in the transfer nodes in the path between the root and $n$ (not including $n$ ). An uncommitted strategy defines a distribution $Z$ over the nodes of the tree (not necessarily leaves) and we can therefore define $Z, U(\theta, \phi)$, optimal strategy and revenue in the exact same way. Notice that every protocol is trivially voluntary for uncommitted buyers, since there is always a strategy guaranteeing the buyer $\max _{a} \mathbb{E}_{\omega}[u(\theta, \omega, a) \mid \theta]$, which is the strategy that defects at any transfer node.

Definition 2.3 We say that it is possible to extract revenue $R$ from a committed (uncommitted) buyer in a context $(u, \mu)$ if there is a voluntary protocol for this context and an optimal committed (uncommitted) strategy $\phi$ for this protocol with revenue at least $R$.

Notice that in the case of committed buyers 'voluntary' is an important restriction, because otherwise, one could simply have a mechanism consisting solely of a transfer node of amount $R$ and a leaf. For uncommitted buyers, however, the only optimal strategy in such a mechanisms would be to defect in the root.

Definition 2.4 We define the optimal revenue that can be extracted from a committed (uncommitted) buyer in a context $(u, \mu)$ to be the maximum $R$ such that for any $\epsilon>0$ it is possible to extract revenue $R-\epsilon$ from a committed (uncommitted) buyer in a context $(u, \mu)$.

\subsection{Revelation Principle and One-Round Revelation Mechanisms}

First we define the concept of a revelation mechanism, in the sense of Gibbard [13] and Myerson [16] and recast their celebrated revelation principle in our setting (its proof is included, for completeness, in Appendix A). For our purposes it would be sufficient to formulate it in terms of revenue (traditional formulations are somewhat more general). 
Definition 2.5 (Revelation Mechanism) A revelation mechanism is a protocol represented by a tree where the root is buyer node, where the buyer is asked to report his type. Moreover, there are no other buyer nodes in the tree. A strategy in such a protocol is truthful if the buyer reports his type truthfully in the root.

Theorem 2.6 (Revelation Principle) Consider any context $(u, \mu)$. If it is possible to extract revenue $R$ from a committed (uncommitted) buyer in the context $(u, \mu)$, then there is a revelation mechanism and a committed (uncommitted) truthful strategy that is optimal for it and produces revenue $R$.

Theorem 2.6 says that we can restrict our attention to Revelation Mechanisms. However, this still allows trees with arbitrary depths and complicated arrangements of seller and transfer nodes. Next we define the stronger notion of One-round revelation mechanisms, where only a very simple interaction between the seller and the buyer is allowed:

Definition 2.7 (One-round Revelation Mechanism) A one-round revelation mechanism is a revelation mechanism represented by a tree of depth three where each path from the root has at most one vertex of each type (i.e. at most one seller node and at most one transfer node).

Now we present formal definitions of two special types of One-round Revelation Mechanisms which were briefly discussed earlier in this section:

Definition 2.8 (Pricing Mappings and Pricing Outcomes) A Pricing Mappings Mechanism is a truthful direct revelation mechanism in which all the children of the root are transfer nodes and all their children are seller nodes. A Pricing Outcomes Mechanism is a truthful direct revelation mechanism in which all the children of the root are seller nodes and all their children are transfer nodes.

\section{Independent signals}

In this section we analyze the case when $\omega$ and $\theta$ are independent, which is simpler than the general case. In this case, the seller's belief about $\theta$ and the buyer's belief about $\omega$ are common knowledge. First we prove that for this setting, we can focus on One-round Revelation Mechanism when searching for the optimal mechanism. Then we show how to compute it efficiently using a convex program. Finally we show that there always exists a protocol with a fairly small tree.

Theorem 3.1 (Existence of a One-Round Optimal Mechanism) Consider any context $(u, \mu)$ such that $\theta$ and $\omega$ are independent. If it is possible to extract revenue $R$ from a committed buyer in the context $(u, \mu)$, then it is possible to extract revenue $R$ from an uncommitted buyer in the same context using a Pricing Mappings Mechanism.

The proof is presented in Appendix B. A consequence of Theorem 3.1 is that with independent signals the fact that the buyer is committed does not help the seller to extract more revenue. In this setting it is possible to extract revenue $R$ from uncommitted buyers if and only if it is possible to extract revenue $R$ from committed buyers.

\section{Pricing Mappings Mechanism.}

Theorem 3.1 allow us to focus on Pricing Mappings Mechanisms. An alternative way of describing a Pricing Mappings Mechanism is as a fixed menu of contracts $\left\{\left(Y_{\theta}, t_{\theta}\right)\right\}_{\theta \in \Theta}$. The contract $\left(Y_{\theta}, t_{\theta}\right)$ 
is intended for a buyer of type $\theta$ (in the sense that it would be optimal for such a buyer to choose that contract out of the menu). A buyer choosing the contract $\left(Y_{\theta}, t_{\theta}\right)$ would pay $t_{\theta}$ and observe one realization of the random variable $Y_{\theta}$ that is correlated with $\omega$, taking values in a finite set $\mathcal{S}_{\theta}$. We call the elements of $\mathcal{S}_{\theta}$ signals, since they reveal to the buyer some information about $\omega$. By buying the contract $\left(Y_{\theta^{\prime}}, t_{\theta^{\prime}}\right)$ a buyer of type $\theta$ gets utility $5 \mathbb{E}_{Y_{\theta^{\prime}}}\left[\max _{a} \mathbb{E}_{\omega}\left[u(\theta, \omega, a) \mid Y_{\theta^{\prime}}\right]\right]-t_{\theta^{\prime}}$. Now, we discuss how to design the menu of contracts in order to maximize revenue.

To be more precise, $Y_{\theta}$ is a random variable that is produced by the seller using $\omega$ and possibly some random bits $r$ that are independent of $(\omega, \theta)$. Without loss of generality we represent $Y_{\theta}$ by a family $\left\{\psi_{\theta}^{\omega}\right\}_{\omega \in \Omega}$ for $\psi_{\theta}^{\omega} \in \Delta\left(\mathcal{S}_{\theta}\right)$. In order to sample $Y_{\theta}$, the seller observes $\omega$ and then samples the value of $Y_{\theta}$ according to $\psi_{\theta}^{\omega}$.

Without loss of generality, we can call $\left(Y_{\theta}, t_{\theta}\right)$ the favorite contract of a buyer of type $\theta$. In order for such set of contracts to be valid we need to make sure that: (1) the protocol is voluntary, i.e., the utility of a buyer of type $\theta$ by taking contract $\left(Y_{\theta}, t_{\theta}\right)$ is at least as high as his utility of not participating in the mechanism and acting using his belief given only $\theta$, and (2) contract $\left(Y_{\theta}, t_{\theta}\right)$ is indeed his favorite one, i.e., he would not strictly prefer to misreport his type and buy a contract $\left(Y_{\theta^{\prime}}, t_{\theta^{\prime}}\right)$ for some $\theta^{\prime} \neq \theta$. Property (1) ensures individual rationality (IR) and property (2) ensures incentive compatibility (IC). Formally:

Definition 3.2 A menu of contracts $\left\{\left(Y_{\theta}, t_{\theta}\right)\right\}_{\theta \in \Theta}$ is valid if and only if:

$$
\begin{aligned}
& \mathbb{E}_{Y_{\theta}}\left[\max _{a} \mathbb{E}_{\omega}\left[u(\theta, \omega, a) \mid Y_{\theta}\right]\right]-t_{\theta} \geq \max _{a} \mathbb{E}_{\omega}[u(\theta, \omega, a)], \quad \forall \theta, \quad\left(I R_{\theta}\right) \\
& \mathbb{E}_{Y_{\theta}}\left[\max _{a} \mathbb{E}_{\omega}\left[u(\theta, \omega, a) \mid Y_{\theta}\right]\right]-t_{\theta} \geq \mathbb{E}_{Y_{\theta^{\prime}}}\left[\max _{a} \mathbb{E}_{\omega}\left[u(\theta, \omega, a) \mid Y_{\theta^{\prime}}\right]\right]-t_{\theta^{\prime}}, \quad \forall \theta \neq \theta^{\prime}, \quad\left(I C_{\theta, \theta^{\prime}}\right)
\end{aligned}
$$

Given a valid menu of contracts, its associated revenue is given by $\sum_{\theta \in \Theta} \mu(\theta) \cdot t_{\theta}$. This definition implicitly assumes that whenever the buyer of type $\theta$ is indifferent between contract $\left(Y_{\theta}, t_{\theta}\right)$ and not buying anything, i.e. the IR constraint is tight, then he buys contract $\left(Y_{\theta}, t_{\theta}\right)$. It also assumes that whenever he is indifferent between $\left(Y_{\theta}, t_{\theta}\right)$ and $\left(Y_{\theta^{\prime}}, t_{\theta^{\prime}}\right)$, he buys $\left(Y_{\theta}, t_{\theta}\right)$. This assumption is without loss of generality, since given any menu of contracts $\left\{\left(Y_{\theta}, t_{\theta}\right)\right\}_{\theta \in \Theta}$ with revenue $R$, for every $\epsilon>0$ it is possible to produce a menu $\left\{\left(Y_{\theta}^{\prime}, t_{\theta}^{\prime}\right)\right\}_{\theta \in \Theta}$ with revenue $(1-\epsilon) \cdot R$ such that all IR and IC inequalities hold strictly. We defer the formal proof of this fact to Lemma C.3.

Our goal is, for any given context, to design the valid menu of contracts with largest possible associated revenue. We call it the optimal menu.

Before starting to optimize the menu, consider a couple of definitions: if $Y_{\theta}$ is a variable taking values in a space $\mathcal{S}_{\theta}$, then for each $s \in \mathcal{S}_{\theta}$, the $Y_{\theta}$-posterior associated with $s$ is the distribution $q \in \Delta(\Omega)$ such that $q(\omega)=\mathbb{P}(\omega \mid s)$. We define the value of buyer of type $\theta$ for posterior $q$ as:

$$
v_{\theta}(q)=\max _{a \in A} \sum_{\omega} q(\omega) u(\theta, \omega, a)
$$

which is a piecewise-linear convex function $v_{\theta}: \mathbb{R}_{+}^{\Omega} \rightarrow \mathbb{R}$. Usually $v_{\theta}(q)$ is defined for $q \in \Delta(\Omega)$, but sometimes it is used for vectors $q \geq 0$ such that $\sum_{\omega} q(\omega) \neq 1$. The reader should note, however that it is a homogeneous function: $v_{\theta}(\lambda q)=\lambda v_{\theta}(q), \forall \lambda>0$.

We next show that we can represent a signal by a distribution over a finite set of posteriors (Observation 3.4) without repetition (Observation 3.3). The proofs of the observations are immediate, but we include in Appendix B for completeness.

Observation 3.3 Given a menu $\left\{\left(Y_{\theta}, t_{\theta}\right)\right\}_{\theta}$, if there are $s, s^{\prime} \in \mathcal{S}_{\theta}$ such that the $Y_{\theta}$-posteriors associated with them are equal, consider the menu obtained by substituting $Y_{\theta}$ by $\tilde{Y}_{\theta}$, where: $\tilde{Y}_{\theta}=s$

\footnotetext{
${ }^{5}$ Since this section talks about the case that $\omega$ and $\theta$ are independent, sampling $\omega \sim \mu(\cdot \mid \theta)$ is the same as sampling $\omega \sim \mu$, and thus in the rest of the section when writing $\mathbb{E}_{\omega}$ we mean $\mathbb{E}_{\omega \sim \mu(\cdot \mid \theta)}$
} 
for $Y_{\theta}=s^{\prime}$ and $\tilde{Y}_{\theta}=Y_{\theta}$ otherwise. The obtained menu is also valid and the revenue associated with it is the same as the one of the original menu.

Given that the posteriors associated with each signal $s \in \mathcal{S}_{\theta}$ are different, we can represent $Y_{\theta}$ by a distribution over a finite set of posteriors, i.e., a set $Q$ of posteriors, each $q \in Q$ being of the form $q \in \Delta(\Omega)$, and a probability $x_{\theta}(q)$ of each posterior $q \in Q$. The condition that a distribution over posteriors represents a random variable correlated with $\omega$ is in the following observation, whose proof is immediate.

Observation 3.4 Let $Y$ be a random variable correlated with $\omega$, i.e., given $\psi^{\omega} \in \Delta(\mathcal{S}), \mathcal{S}=[k], Y$ is sampled by first sampling $\omega \sim \mu$ and then sampling from $\psi^{\omega}$. Consider a set $Q=\left\{q_{1}, \ldots, q_{k}\right\} \subset$ $\Delta(\Omega)$ and $x \in \Delta(Q)$. The pair $(Q, x)$ represent a distribution over posteriors of a random variable $Y$ correlated with $\omega$ if and only if:

$$
\sum_{q_{i} \in Q} x\left(q_{i}\right) \cdot q_{i}(\omega)=\mu(\omega) \quad \forall \omega \in \Omega
$$

From this point on, we represent each $Y_{\theta}$ as a function $x_{\theta}: \Delta(\Omega) \rightarrow[0,1]$ with finite support, satisfying Equation $(F)$ for each $\theta$.

$$
\sum_{q_{i} \in Q} x_{\theta}\left(q_{i}\right) \cdot q_{i}(\omega)=\mu(\omega) \quad \forall \omega \in \Omega,
$$

For any finite set of posteriors $Q \subset \Delta(\Omega)$, we can formulate a restricted revenue maximization problem - for mechanisms that offer a menu of contracts with posteriors restricted to belong to $Q$ - as $\mathbf{L P}_{\mathbf{1}}$ below with variables $x_{\theta}(q)$ for each $\theta \in \Theta, q \in Q$ and $t_{\theta}$ for each $\theta \in \Theta$. Recall that $p \in \Delta(\Omega)$ is the prior on $\omega$, i.e., $p(\omega)=\mu(\omega)$.

$$
\begin{aligned}
& \mathbf{L P}_{\mathbf{1}}: \max \sum_{\theta} \mu(\theta) t_{\theta} \text { s.t. } \\
& \sum_{q} v_{\theta}(q) x_{\theta}(q)-t_{\theta} \geq v_{\theta}(p), \quad \forall \theta, \quad\left(I R_{\theta}\right) \\
& \sum_{q} v_{\theta}(q) x_{\theta}(q)-t_{\theta} \geq \sum_{q} v_{\theta}(q) x_{\theta^{\prime}}(q)-t_{\theta^{\prime}}, \quad \forall \theta^{\prime} \neq \theta, \quad\left(I C_{\theta, \theta^{\prime}}\right) \\
& \sum_{q} x_{\theta}(q) \cdot q(\omega)=\mu(\omega) \quad \forall \theta, \omega, \quad\left(F_{\theta}\right) \\
& x_{\theta}(q), t_{\theta} \geq 0, \quad \forall \theta, q
\end{aligned}
$$

The constraints in $\mathbf{L P}_{\mathbf{1}}$ correspond to the characterization of valid contracts in Definition 3.2 and the feasibility of the representation of contracts as distributions over posteriors in Observation 3.4.

To work with a linear program of finite size, we have restricted $q$ to belong to a finite set $Q$. It is conceptually easier to think of $q$ as ranging over the entire set $\Delta(\Omega)$, in which case $\mathbf{L P}_{\mathbf{1}}$ would represent the revenue maximization problem in full generality. The following lemma, whose proof is in the appendix, shows that the restriction to a finite set of posteriors is without loss of generality. There is a finite set $Q^{*}$ that can be precomputed from knowledge of the function $u$ alone, such that solving $\mathbf{L} \mathbf{P}_{\mathbf{1}}$ with $Q=Q^{*}$ is guaranteed to produce an optimal menu of contracts.

Lemma 3.5 (Interesting Posteriors) Given $u: \Theta \times \Omega \times A \rightarrow \mathbb{R}$, there is a finite set $Q^{*} \subset \Delta(\Omega)$ such that for all $\mu$, the maximum revenue that can be extracted by any protocol can also be extracted by a protocol that is limited to use posteriors in $Q^{*}$. Moreover, all the elements $q \in Q^{*}$ can be represented with polynomially many bits.

By passing to the dual of $\mathbf{L P}_{\mathbf{1}}$, we get an $\mathrm{LP}$ with $O\left(|\Theta|^{2}\right)$ variables. Below, we give a separation oracle for the dual showing that it can be solved in polynomial time. The variables of the dual are $g_{\theta}, h_{\theta, \theta^{\prime}} \in \mathbb{R}$ and $y_{\theta} \in \mathbb{R}^{\Omega}$ : 


$$
\begin{aligned}
& \mathbf{D L P}_{1}: \min \sum_{\theta} p^{t} y_{\theta} \text { s.t. } \\
& -v_{\theta}(q) g_{\theta}+\sum_{\theta^{\prime} \neq \theta}\left[v_{\theta^{\prime}}(q) h_{\theta^{\prime}, \theta}-v_{\theta}(q) h_{\theta, \theta^{\prime}}\right]+q^{t} y_{\theta} \geq 0, \quad \forall \theta, q \\
& g_{\theta}+\sum_{\theta^{\prime} \neq \theta}\left[h_{\theta, \theta^{\prime}}-h_{\theta^{\prime}, \theta}\right] \geq \mu(\theta), \quad \forall \theta \\
& g_{\theta}, h_{\theta, \theta^{\prime}} \geq 0, y_{\theta} \in \mathbb{R}^{\Omega}, \quad \forall \theta, \theta^{\prime}
\end{aligned}
$$

Separation oracle: The second family of constraints is of size $|\Theta|$, so separating it is trivial. In order to separate the first family we re-write the constraints in a different way. Notice that the $v_{\theta}(\cdot)$ is the maximum over $|A|$ linear functions. So, we can substitute each constraint of the first family for the following $|A|$ constraints:

$$
\sum_{\theta^{\prime} \neq \theta} v_{\theta^{\prime}}(q) h_{\theta^{\prime}, \theta} \geq-q^{t} y_{\theta}+\left(g_{\theta}+\sum_{\theta^{\prime} \neq \theta} h_{\theta, \theta^{\prime}}\right) \sum_{\omega} u(\theta, \omega, a) q(\omega)
$$

for all $a \in A, \theta \in \Theta$ and $q \in Q^{*}$. Now, for fixed $a, \theta$ we want to check if this constraint is satisfied by all $q \in Q^{*}$ or find one $q$ for which the constraint is violated.

Relaxing the requirement $q \in Q^{*}$ to $q \in \Delta(\Omega)$, this is equivalent to solving the following convex programming problem:

$$
\min _{q \in \Delta(\Omega)} \sum_{\theta^{\prime} \neq \theta} v_{\theta^{\prime}}(q) h_{\theta^{\prime}, \theta}+q^{t} y_{\theta}-\left(g_{\theta}+\sum_{\theta^{\prime} \neq \theta} h_{\theta, \theta^{\prime}}\right) \sum_{\omega} u(\theta, \omega, a) q(\omega)
$$

Lemma 3.6 The convex programming problem above can be solved exactly in polynomial time. Given an optimal solution $q \in \Delta(\Omega)$, there must exist another optimal solution $q^{*}$ that belongs to $Q^{*}$, and we can find such a $q^{*}$ in polynomial time.

The proof (given in the appendix) assumes that the set $A$ is finite and polynomially bounded.

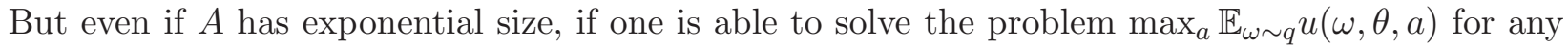
posterior $q$, we can still solve the problem.

Comparison with Sealed Envelope. The sealed envelope mechanism presented in Section 2.1 can extract at least $1 /|\Theta|$ revenue of the optimal mechanism, by the following simple observation: if a there is a voluntary protocol that extracts revenue $R$ from a certain context $(u, \mu)$, then there is at least one $\theta$ for which $\frac{R}{\Theta \Theta} \leq \mu(\theta) \cdot \xi(\theta)$, where $\xi(\theta)=\mathbb{E}_{\omega}\left[\max _{a} u(\theta, \omega, a)\right]-\mathbb{E}_{\omega}\left[\max _{a} u(\theta, \omega, a)\right]$ is the maximum surplus that can be extracted from a buyer of type $\theta$. By setting the price of the envelope to $t=\xi(\theta)-\epsilon$ for some tiny $\epsilon>0$, the mechanism guarantees revenue at least $\mu(\theta) \cdot \xi(\theta)$. In Example B.1 we show this bound is tight by presenting a context where the sealed envelope mechanism can not extract more then $\Omega\left(\frac{1}{|\Theta|}\right)$ of the optimal mechanism.

Protocols with Small Trees. We just showed how to compute the revenue optimal protocol in polynomial time when $\theta$ and $\omega$ are independent. We know that the protocol has polynomial size, where its size is measured by the number of nodes in the tree representing the protocol. Here, we make it more explicit and show that there is a protocol of size $O\left(|\Omega| \cdot|\Theta|+|\Theta|^{2}\right)$. We show that by bounding the sum of the support size of the random variables in the menu of contracts. This bounds the number of leaves of the One-round Revelation Mechanism (Theorem 3.1). As the number of nodes in the tree representing this protocol is at most 3 times the number of leaves (twice the number of leaves plus one node for the buyer and $|\Theta|$ seller nodes), the bound on the size of the tree follows from the bound on the number of leaves. The proof of the next theorem is in Appendix B.

Theorem 3.7 Let $n=|\Theta|$ and $m=|\Omega|$. Denote the support of a vector $x$ by $\|x\|_{0}$. The program $\mathbf{L P}_{1}$ has a solution where $\left\|x_{\theta}\right\|_{0} \leq m+n-1$ for all $\theta$, and $\sum_{\theta}\left\|x_{\theta}\right\|_{0} \leq m n+\left(\begin{array}{l}n \\ 2\end{array}\right)$. Moreover, there are settings in which support of size quadratic in $n$ is necessary, even when $m=2$. 
The fact that the quadratic lower bound holds even for $m=2$ (Example B.2) is somewhat counterintuitive: even when the information being sold is a single bit, there are contexts in which revenue maximization requires using signals that consist of $\Omega(\log n)$ bits.

\section{Correlated signals with committed buyers}

In Section 3 we have considered independent signals and observed that for that case the seller does not care if the buyer is committed or not. In this section we consider correlated signals and committed buyers, the case of correlated signals and uncommitted buyers will be discussed in Section 5. Throughout this section we assume that the buyer is committed.

\subsection{Pricing Outcomes Mechanism}

In the previous section we showed that if $\theta, \omega$ are independent, then the optimal protocol had the form of offering a menu of contracts with a fixed price for each contract. In this section we show that this is not sufficient to optimize the revenue whenever $\theta, \omega$ are correlated. In order to optimize revenue, we need to add a twist: we still offer a menu of options, each option having a random variable $Y_{\theta}$ correlated with $\omega$ and taking values in $\mathcal{S}_{\theta}$. Instead of a fixed price, however, we charge a specific price for each outcome $s \in \mathcal{S}_{\theta}$ of the signaling scheme. We continue to refer to the options on the menu as contracts, although this means that the word contracts has a slightly different meaning in this section than in the preceding one (as it is no longer the case that the buyer pays before observing any signal).

Why does this construction help in designing mechanisms to optimize revenue? Suppose a seller designs a variable $Y$ taking values in $\mathcal{S}$. Consider $\psi^{\omega} \in \Delta(\mathcal{S})$ for each $\omega$ such that the seller produces $s \in \mathcal{S}$ by sampling $s$ according to $\psi^{\omega}$. If $\omega$ and $\theta$ were independent, the seller would be always choosing the same distribution from the buyer's perspective, which would be $\mathbb{P}(s)=\sum_{\omega} \mu(\omega) \cdot \mathbb{P}\left(\psi^{\omega}=s\right)$. However, since $\theta$ and $\omega$ are correlated, different buyer-types perceive different distributions over $\mathcal{S}$ : a buyer of type $\theta$ perceives $\mathbb{P}(s \mid \theta)=\sum_{\omega} \mu(\omega \mid \theta) \cdot \mathbb{P}\left(\psi^{\omega}=s\right)$. So, if we condition the prices on the outcomes $s$, two different buyer-types see different prices for the same contract. This increases our power of price-discrimination. For the case of committed buyers we are able to show the existence of an optimal One-Round Revelation Mechanism.

Theorem 4.1 (Existence of a One-Round Optimal Mechanism) For any context $(u, \mu)$, if it is possible to extract revenue $R$ from a committed buyer in this context, then there is a Pricing Outcomes Mechanism that does so.

The proof, which is given in Appendix $\mathrm{C}$, is essentially the same as the proof of Theorem 3.1. except for the last step. Previously this step relied on the independence of $\theta$ and $\omega$; here, we instead rely on the fact that seller nodes are situated above transfer nodes in our protocol, which eliminates the need to estimate expected transfers over a random execution of the original protocol, and instead lets us match transfers pointwise.

It should be noted that the fact that buyers are committed to follow the mechanism until the end is crucial. In fact, in any protocol containing a transfer node in which the buyer needs to pay the seller and whose child is a leaf, the optimal uncommitted strategy would be to defect at that transfer node. In other words, uncommitted buyers could acquire the information and leave without paying. We mention one way to solve this problem: before the mechanism starts, ask a large sum of money from the buyer. Run the mechanism and then gives the large sum of money back to the buyer. This will guarantee that the buyer follows the mechanism until the end, so as not to lose his 
initial deposit. This will add an extra level to the mechanism: one transfer node in the beginning to charge this large sum of money. The rebate in the end can simply blend with the last transfer.

Pricing Outcomes Mechanism. We will describe a Pricing Outcomes Mechanism using the following notation. The seller designs a menu of contracts solely based on the context. The menu is a collection $\left\{\left(Y_{\theta}, t_{\theta}\right)\right\}_{\theta \in \Theta}$ where $Y_{\theta}$ is a random variable correlated with $\omega$ and taking values in a finite set $\mathcal{S}_{\theta}$, just like in the independent case. The payment function, however, is a function $t_{\theta}: \mathcal{S}_{\theta} \rightarrow \mathbb{R}$ and it allows for both positive and negative payments. The seller outputs $s \sim Y_{\theta}, t_{\theta}(s)$, which is a signal and a payment request of $t_{\theta}(s)$. If $t_{\theta}(s)<0$, the seller transfers $\left|t_{\theta}(s)\right|$ to the buyer.

In the following, we discuss how to find the menu maximizing revenue using a convex program. The derivation of this convex program closely parallels the derivation of the corresponding convex program in the independent case. We give the full details in Appendix $\mathbb{C}$, and here we limit ourselves to discussing the two most salient differences between the derivation of the convex program in the independent and correlated cases.

(1) The posterior vector after receiving a given signal depends upon the buyer's type. To compare posteriors across different types, we adopt a common "frame of reference" - that of an outside observer who observes the signal sent by the seller but does not observe $\theta$ - and we translate from the type- $\theta$ reference frame to the outside observer's reference frame using a matrix $D_{\theta}$ that expresses Bayes' rule.

(2) Since payments are now associated with signals, the obvious way of expressing the expected revenue is as a sum of products, where each term is the probability of sending a particular signal, $x_{\theta}(q)$, multiplied by the amount charged in the event of sending it, $t_{\theta}(q)$. Thus, our program has a quadratic objective function if we treat both the probability and the transfers as primal variables. (This issue does not arise in Pricing Mappings, since a buyer of type $\theta$ pays the same amount regardless of what signal is sent, hence the variables $x_{\theta}(q)$ do not appear in the objective function.) To make the objective function linear when pricing outputs, we define new variables $\tilde{t}_{\theta}(q)=x_{\theta}(q) \cdot t_{\theta}(q)$. Fortunately, this change of variables makes the constraints linear as well.

Full Surplus Extraction. Correlation can be very valuable to the seller. In fact, if the distribution exhibits sufficiently complex correlation, the seller might be able to extract full surplus from the buyers using a Pricing Outcomes Mechanism. Given a context $(u, \mu)$ we define the full surplus as the expected gain the buyer would get by learning the value of $\omega$. In other words, the full surplus is $\mathbb{E}_{\theta}[\xi(\theta)]$, where $\xi(\theta)=\mathbb{E}\left[\max _{a} u(\theta, \omega, a) \mid \theta\right]-\max _{a} \mathbb{E}[u(\theta, \omega, a) \mid \theta]$. Clearly no mechanism can extract more then the full surplus, and extracting a $1 /|\Theta|$ fraction of it is trivial, even using a sealed envelope mechanism, as was observed in Section 3. Now, we show that if $\mu$ is sufficiently correlated, then we can extract the full surplus. Our result leverages the ideas developed by Cremer and McLean [9, 10] in their work on auctions with correlated bidders, although obviously the setting in which we apply these ideas is different from theirs.

For a joint distribution $\mu$ over $\Omega \times \Theta$ we define $\operatorname{rank}(\mu)$ as the rank of the $|\Omega| \times|\Theta|$ matrix defined by $\mu(\omega, \theta)$. For example, if $\omega$ and $\theta$ are independent, then $\operatorname{rank}(\mu)=1$.

Theorem 4.2 If $\operatorname{rank}(\mu)=|\Theta|$ then the optimal Pricing Outcomes Mechanism extracts the full surplus. Moreover, this can be done with a single contract.

Proof : We define one single contract in the following way. Calculate $t \in \mathbb{R}^{\Omega}$ such that:

$$
\sum_{\omega \in \Omega} t(\omega) \cdot \mu(\omega, \theta)=\mu(\theta) \cdot \xi(\theta), \forall \theta
$$


Since $\operatorname{rank}(\mu)=|\Theta|$, this system is guaranteed to have a feasible solution. Now, offer the following contract to all the buyers: the seller reveals the value of $\omega$ and requests a payment of $t(\omega)$. By the definition of $t$, each buyer is indifferent between buying this contract or not buying anything, so the mechanism is voluntary.

Notice that one can, in the manner of Lemma C.3, offer the above contract with price $(1-\epsilon) \cdot t(\omega)$ for each outcome, getting revenue arbitrarily close to the full surplus and making the players strictly prefer to buy the contract.

At this point it is instructive to consider a concrete example for full surplus extraction.

Example 4.3 Imagine a box that has a locker on it and there are two keys labeled with 0 and 1 , exactly one of which can open the box. The buyer can choose one key and try it. If he opens the box, he gets the object inside. Let the type of the buyer $\theta$ encode his value $z_{\theta}$ for the object and some signal that gives him a hint of which might be the right key. The seller knows exactly what is the correct key, and let this be $\omega$. How should the seller sell the information to the buyer?

Consider $\Omega=A=\{0,1\}, \Theta=[2]$ and the reward function as $u(\theta, \omega, a)=z_{\theta} \cdot \mathbb{1}\{\omega=a\}$, with $z_{1}=3, z_{2}=5$. The joint distribution is $[\mu(\omega, \theta)]=\left[\begin{array}{lll}0.2 & 0.3 \\ 0.3 & 0.2\end{array}\right]$.

Before participating in the mechanism a buyer of type $\theta=1$ has interim belief $(0.4,0.6)$ on $\omega$, so his best action is to pick key 1 , getting expected reward $0.6 \cdot 3=1.8$. If he is able to pick key 0 whenever $\omega=0$ and key 1 whenever $\omega=1$, then he always get a reward of 3 , so his value for the information is $\xi(1)=3-1.8=1.2$. Similarly $\xi(2)=2$. In order to design a contract that extracts full surplus, find $t(\omega)$ such that:

$$
0.4 \cdot t(0)+0.6 \cdot t(1)=\xi(1) \quad \text { and } \quad 0.6 \cdot t(0)+0.4 \cdot t(1)=\xi(2)
$$

Solving this system, we get: $t_{1}(0)=3.6, t_{1}(1)=-0.4$. This means that if the seller reveals signal $\omega=0$, the buyer needs to pay 3.6, if the seller reveals $\omega=1$, the buyer receives 0.4 from the seller. Both buyer types see the full information contract being offered, but because of the correlation of $\theta$ and $\omega$, they perceive its expected cost to be different. Player of type $\theta$ perceives the expected cost to be $\mathbb{E}[t(\omega) \mid \theta]=t(0) \mu(\omega=0 \mid \theta)+t(1) \mu(\omega=1 \mid \theta)$. This feature of correlation gives the seller a great power to do price discrimination.

\subsection{Continuity and Approximation}

One might be tempted to conclude from Theorem 4.2 that for any distribution $\mu$ such that $|\Theta| \leq|\Omega|$, one is able to extract full surplus since all matrices $[\mu(\omega, \theta)]$ can be approximated arbitrarily closely by matrices of full rank. The flaw in this argument is obvious. If one sees $\mu$ as a $\Theta \times \Omega$ matrix, and $\xi \in \mathbb{R}^{\Theta}$ as a vector with the surplus $\xi(\theta)$ in component $\theta$, the payment vector $t \in \mathbb{R}^{\Omega}$ in Theorem 4.2 can be found by solving the linear system $\mu \cdot t=\xi$. If $\mu$ is a perturbation of, say, a rank-one matrix (corresponding to $\theta$ and $\omega$ being independent) then the linear system is very ill-conditioned, and therefore the solution $t$ has a very high norm. This causes $t$ to diverge as $\mu$ becomes closer to being independent. To illustrate this point, let us revisit Example 4.3 ,

Example 4.4 Consider the same reward function as in Example 4.3 but with a different probability distribution: $\Omega=A=\{0,1\}, \Theta=[2], u(\theta, \omega, a)=z_{\theta} \cdot \mathbb{1}\{\omega=a\}$, with $z_{1}=3, z_{2}=5$, and distribution $[\mu(\omega, \theta)]=\left[\begin{array}{ll}0.25 & 0.25 \\ 0.25 & 0.25\end{array}\right]$.

The surplus of each buyer is given by $\xi(1)=1.5, \xi(2)=2.5$. Proceeding as described in Section 3. one finds that the optimal mechanism is to offer one single contract that outputs $\omega$ exactly and costs 1.5. Clearly both types buy this contract and the expected revenue is 1.5. Notice however that the expected surplus is $\frac{1}{2} \xi(1)+\frac{1}{2} \xi(2)=2$ and Theorem 4.2 guarantees that for a slightly 
perturbed joint distribution one can extract it entirely. For example, consider $\mu=\left[\begin{array}{lll}0.25001 & 0.24999 \\ 0.24999 & 0.25001\end{array}\right]$. Applying the proof of Theorem 4.2 we get the following mechanism extracting revenue 2: offer the contract that outputs the full information and if the outcome is $\omega$, a player pays $t(\omega)$ where $[t(0), t(1)]=[-24996,25004]$.

This example highlights two problems with the optimal mechanism. The most obvious is that it somehow abuses risk-neutrality. The optimal mechanism produces very large payments which are balanced by large rebates. This situation is clearly not desirable in practice. The second problem is that the revenue that can be extracted from a certain context might change abruptly whenever the context changes slightly.

It turns out that these discontinuities in the optimal-revenue function are only unidirectional: as one varies the context, the revenue can abruptly decrease but it cannot abruptly increase. Furthermore, for certain restricted classes of mechanisms, the optimal revenue depends continuously on the context. In particular, this holds for the first three members of the following sequence of progressively more general types of mechanisms 6

$$
\left\{\begin{array}{c}
\text { Sealed } \\
\text { Envelope }
\end{array}\right\} \subseteq\left\{\begin{array}{c}
\text { Pricing } \\
\text { Mappings }
\end{array}\right\} \subseteq\left\{\begin{array}{c}
\text { Pricing Outcomes } \\
t \geq 0
\end{array}\right\} \subseteq\left\{\begin{array}{c}
\text { Pricing } \\
\text { Outcomes }
\end{array}\right\}
$$

To formalize these continuity assertions, let us fix $\Omega, \Theta$, and $A$, and regard a context $(u, \mu)$ as a point in the topological space $\mathcal{C}=\mathbb{R}^{\Theta \times \Omega \times A} \times \Delta(\Omega \times \Theta)$ equipped with its standard topology. The revenue of the optimal mechanism for committed buyers (or, equivalently, the optimal Pricing Outcomes Mechanism) will be denoted by $R(u, \mu)$. Similarly, we use $R_{e}, R_{c}, R_{p}$ respectively to denote the revenue of the optimal Sealed Envelope Mechanism, Pricing Mappings Mechanism, or Pricing Outcomes Mechanism with No Positive Transfers. The following theorem formalizes the continuity assertions claimed above. Recall that a function $f: \mathbb{R}^{k} \rightarrow \mathbb{R}$ is lower-semicontinuous if for all converging sequences $x_{i} \rightarrow x, f(x) \leq \liminf f\left(x_{i}\right)$.

Theorem 4.5 The function $R: \mathcal{C} \rightarrow \mathbb{R}$ is lower-semicontinuous. The functions $R_{e}, R_{c}: \mathcal{C} \rightarrow \mathbb{R}$ are continuous. Let $\mathcal{C}^{\circ}$ be the set of contexts $(u, \mu)$ such that $\mu(\omega, \theta)>0$ for all $\omega, \theta$ (henceforth called non-degenerated contexts), the function $R_{p}: \mathcal{C}^{\circ} \rightarrow \mathbb{R}$ is continuous.

Appendix D.1 breaks the theorem down into pieces and proves each piece. The fact that $R$ is lowersemicontinuous is proven by appealing to Lemma C.3 which shows that for any context $(u, \mu)$, the optimal revenue can be approximated arbitrarily closely by mechanisms in which all of the IR and IC constraints are slack. Such a mechanism remains valid in a neighborhood of $(u, \mu)$, and its revenue varies continuously in this neighborhood, hence the optimal revenue throughout a neighborhood of $(u, \mu)$ remains nearly as great as the optimal revenue at $(u, \mu)$. As a side effect of the method of proof, we obtain a robustness result: if the seller believes that the context $(u, \mu)$ and designs an protocol extracting $R(u, \mu)$, but the real context may actually be slightly different, the seller can design a slightly different protocol extracting revenue at least $(1-\epsilon) \cdot R(u, \mu)$ from any context $\left(u^{\prime}, \mu^{\prime}\right)$ that is sufficiently close to $(u, \mu)$. We note that a proof in the same spirit for the Cremer and McLean setting can be found in Robert [19]. Notice that the heart of our proof is the application of Lemma C.3. In this specific ingredient, our proof is different from that of Robert.

\footnotetext{
${ }^{6}$ A protocol has no positive transfers if at any transfer node $n$ the seller never pays the buyer, i.e. $t(n) \geq 0$. We discuss Pricing Outcomes Mechanism with No Positive Transfers as this mechanism achieves the optimal revenue for committed buyers with no positive transfers. (This follows from the proof of Theorem 4.1)

Note that we use the term no positive transfers as it is used in literature on auction theory, to exclude mechanisms in which the seller pays money to the buyer. We chose to use this standard term although in our setting such a payment is represented by a transfer node with negative transfer $t(n)<0$.
} 
The same argument establishes lower-semicontinuity of the functions $R_{e}, R_{c}, R_{p}$. Their uppersemicontinuity follows from a compactness argument. For each of these classes of mechanisms, the optimal revenue can be found by solving a variant of $\mathbf{L P}_{\mathbf{2}}$ that reflects the additional constraints on the mechanism. Each such variant LP has an unbounded feasible region, and the first step of the proof is to identify a compact (i.e. closed and bounded) subset of the feasible region that is guaranteed to contain the optimal LP solution, at least for all contexts in a neighborhood of a given $(u, \mu)$. Taking any sequence of contexts $\left(u_{i}, \mu_{i}\right)$ converging to $(u, \mu)$ and passing to a subsequence if necessary, we may assume that their optimal mechanisms $\mathcal{M}_{i}$ converge to a limit $\mathcal{M}$ belonging to the feasible region of the LP. This mechanism $\mathcal{M}$ then supplies a lower bound on the optimal revenue in context $(u, \mu)$ that suffices to prove upper-semicontinuity.

Approximating Revenue. We have seen that the Sealed Envelope Mechanism achieves at least a $(1 / n)$-approximation to the optimal revenue, where $n=|\Theta|$, and that this bound cannot be improved by more than a constant factor in the worst case. The other two classes of mechanisms listed above - Pricing Mappings, and Pricing Outcomes without positive transfers - are substantially simpler and more natural than Pricing Outcomes in full generality, so it would be desirable to approximate the optimal revenue using one of these simpler classes of mechanisms. Unfortunately, in the worst case, the approximation achieved is rather poor: we prove in Appendix D.2 that there exist contexts $(u, \mu)$ such that $R_{c}(u, \mu) \leq R_{p}(u, \mu) \leq O(1 / n) \cdot R(u, \mu)$. The proof is an application of Theorem 4.5. We carefully construct a context with independent $\omega, \theta$ for which the full surplus exceeds $R_{c}(u, \mu)$ by a factor of $\Omega(n)$. For such a context Pricing Mappings is optimal, i.e. $R_{c}(u, \mu)=R_{p}(u, \mu)=R(u, \mu)$. Now if we slightly perturb $\mu$ to make it into a full-rank matrix, Theorem 4.2 tells us that $R(u, \mu)$ jumps up by a factor of $\Omega(n)$ to match the full surplus, while Theorem 4.5 ensures that $R_{c}(u, \mu)$ and $R_{p}(u, \mu)$ can only change by a tiny amount.

Finally there remains the question of whether $R_{c}$ achieves a good approximation to $R_{p}$ in the worst case. As it happens, once again the worst-case ratio between these two quantities is $\Omega(n)$, as evidenced by Example D.7 in the Appendix. In this example the seller gets from a side channel the information about the the buyer's surplus. In a Pricing Outcomes Mechanism (even without positive transfers), the seller is capable of leveraging this information, while in a Pricing Mappings Mechanism, she is not.

\section{Correlated Signals with Uncommitted Buyers}

In this section we examine mechanisms for uncommitted buyers when signals are correlated, and we formulate an intriguing open question related to a surprising failure of the one-round revelation property. First, we review what is known about uncommitted buyers from previous sections. Theorem 3.1 says that if $\omega$ and $\theta$ are independent, the revenue-optimal mechanism is aPricing Mappings Mechanism. The optimal strategy for both committed and uncommitted buyers is the same in such a mechanism. However, when $\omega$ and $\theta$ are correlated, the revenue-optimal mechanism for committed buyers is a Pricing Outcomes Mechanism (Theorem 4.1). In such a mechanism, the buyer declares his type, the seller sends a signal and the buyer pays a certain amount of money that depends on the signal sent by the seller. Such a mechanism clearly does not work for uncommitted buyers, who can defect after getting the signal but before the payment.

In section 4.1, we mentioned one way to get around this problem: before executing the Pricing Outcomes Mechanism the seller can require the buyer to deposit a large amount of money, then the mechanism executes, and after it completes the seller refunds the buyer. This mechanism has clear practical drawbacks: in practice a deposit-refund scheme increases the cost of participation as it requires the buyer to always be able to make large payments, even in cases in which at the end 
of the protocol the net payment is small, or no payments are made in the execution. The latter case is particularly problematic as it is usually costly to establish a payment relationship (e.g. the buyer needs to spend time giving his credit card information) so always imposing payments might deter some buyers.

A protocol has no positive transfers if at any transfer node money always goes from the buyer to the seller, i.e. $t(n) \geq 0$ for every transfer node $n$. Once we exclude positive transfers the problem of designing optimal mechanisms for uncommitted buyers becomes quite challenging. We formulate it as the following open problem:

Open Problem 5.1 Characterize the protocols that extract maximum revenue from uncommitted buyers subject to no positive transfers. In particular, is it possible to design an algorithm that decides, given any context $(u, \mu)$ and target revenue $R$, whether there exists a protocol with no positive transfers that extracts revenue $R$ from uncommitted buyers?

A natural first attempt to address this problem would be to prove, in the manner of Theorem 3.1 and Theorem 4.1, that for this setting one can extract optimal revenue using a One-Round Revelation Mechanism. We show that this approach fails.

Theorem 5.2 (Failure of the One-round Revelation Property) There exists a context $(u, \mu)$ with correlated $\omega$ and $\theta$ for which some generic protocol with no positive transfers extracts strictly more revenue from uncommitted buyers than any One-round Revelation Mechanism with no positive transfers.

In order to prove this theorem, we observe that if it is possible to extract revenue $R$ from an uncommitted buyer using a One-round Revelation Mechanism, then it is possible to extract the same revenue using a Pricing Mappings Mechanism. This follows easily from the fact that if a transfer node $n$ is the last node before the leaf in a path of the protocol tree, an uncommitted buyer will always defect before this node if $t(n)>0$.

Then in Example 5.3 we present a context for which an interactive protocol with no positive transfers extracts strictly more revenue than any Pricing Mappings Mechanism.

Example 5.3 We present a context $(u, \mu)$ for which a mechanism where the seller interacts with the buyer twice (producing a protocol tree of height 4) extracts strictly more revenue than any direct revelation mechanism.

Let $\Omega=\{0,1\}, \Theta=\{0,1\}$ and the distribution $\mu=\left[\begin{array}{ll}0.3 & 0.2 \\ 0.2 & 0.3\end{array}\right]$. Let $A=\{0,1\}$ and define the utility such that $u(\theta, \omega, 0)=\omega$ (for $a=0$ ) and $u(\theta, \omega, 1)=1-9 \omega$ (for $a=1$ ). As usual, since $\Omega=\{0,1\}$ we represent the posterior probability by one real number $q \in[0,1]$, the probability of the event $\omega=1$. The first part of Figure 2 depicts the utility as a function of the posterior $q$, the utility is represented by functions $v_{\theta}:[0,1] \rightarrow \mathbb{R}$. In this particular case $v_{0}(\cdot)=v_{1}(\cdot)=v(\cdot)$.

It is simple to see that the optimal Pricing Mappings Mechanism offers a single contract, pricing the full information (value of $\omega$ ) at 0.4 , and getting revenue of 0.4 .

We next present a protocol with no positive transfers that extracts strictly more revenue than the optimal Pricing Mappings Mechanism from uncommitted buyers. The protocol is represented by a tree of height 4 depicted in the second part of Figure Q. It consists of two transfer nodes with amounts $t_{1}=0.533$ and $t_{2}=0.8$. For the seller nodes, the transition probabilities are as follows:

- node $\mathbf{s}_{1}$ : the seller outputs $\ell_{1}$ whenever $\omega=0$ and outputs $\ell_{2}$ whenever $\omega=1$.

- node $\mathbf{s}_{3}$ : the seller outputs $\ell_{3}$ whenever $\omega=0$ and outputs $\ell_{4}$ whenever $\omega=1$. 

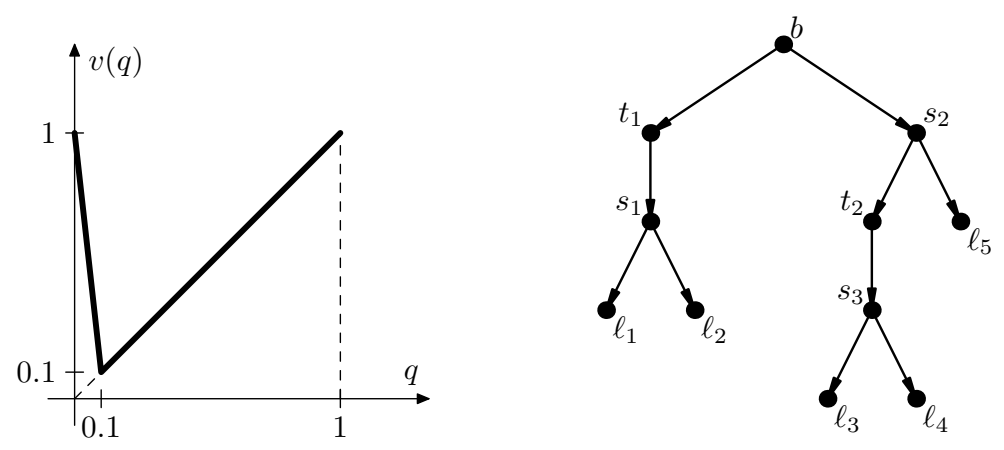

Figure 2: Example 5.3, a protocol for uncommitted buyers generating strictly more revenue then any direct-revelation-mechanism.

- node $\mathbf{s}_{2}$ : the seller moves either to node $t_{2}$ or to node $\ell_{5}$ according to the following probabilities: $\mathbb{P}\left(\ell_{5} \mid \omega=0\right)=0, \mathbb{P}\left(\ell_{5} \mid \omega=1\right)=5 / 6$ and clearly $\mathbb{P}\left(t_{2} \mid \omega\right)=1-\mathbb{P}\left(\ell_{5} \mid \omega\right)$.

Now, we claim that the optimal strategy for an uncommitted buyer is to play left (to node $t_{1}$ ) whenever $\theta=0$ and play right (to node $s_{2}$ ) whenever $\theta=1$, and then follow the protocol (make the transfers when asked) without defecting. We prove this in Claim E.1 in Appendix E. Given these strategies we calculate the expected revenue of the protocol, which is: $\mu(\theta=0) \cdot 0.533+\mu(\theta=$ 1) $\cdot 0.8 \cdot \mathbb{P}\left(t_{2} \mid \theta=1\right)=0.4665>0.4$

We have shown above that generic protocols can extract strictly more revenue than the Oneround Revelation Mechanism when buyers are uncommitted and no positive transfers are allowed. One might wonder if such interactive mechanisms can extract as much revenue from uncommitted buyers as can be extracted from committed buyers. We show that the answer is no, as for the setting of Example 5.3 there is a gap between the two.

Theorem 5.4 There exists a context with correlated $\omega$ and $\theta$ for which the optimal revenue that can be extracted from uncommitted buyers using a protocol with no positive transfers is strictly less than the optimal revenue that can be extracted from committed buyers.

We prove the theorem in Appendix E. For context $(u, \mu)$, the optimal revenue that can be extracted from committed buyers using protocols that has no positive transfers is exactly the revenue $R_{p}(u, \mu)$ that can be extracted by Pricing Outcomes with No Positive Transfers Mechanism. To prove the claim we show in Appendix E that for the setting of Example 5.3 for some $\delta>0$ it is impossible to extract revenue of $R_{p}(u, \mu)-\delta$ from uncommitted buyers using protocols that have no positive transfers.

The intuition behind the proof of Theorem 5.4 is the following: in this context, it is possible to extract the full surplus from committed buyers. In order to extract close to this much revenue from uncommitted buyers, the mechanism must be offering an option that results with some posterior very close to the full information. Now we can show that since a buyer of $\theta=1$ is paying at most his entire surplus, there is a deviation for a buyer of type $\theta=0$ that guarantees him almost full information for a price considerably below his surplus.

\section{Open Problems}

We believe the design of mechanisms for selling information is an area full of exciting possibilities. In particular, there might be potential connections with information theory and cryptography, that 
could be discovered and formalized. In this section we describe some interesting open directions, listing them from the more precise to the more vague problems:

1. Mechanisms for Uncommitted Buyers: The main open problem left by our paper is the problem of designing revenue optimal protocols for uncommitted buyers, when no positive transfers are allowed (Open Problem 5.1). Theorem 5.2 reveals that multiple rounds of partial information disclosure (interleaved by payment to the seller) are sometimes necessary to achieve optimal revenue if the buyer is allowed to abort his interaction with the seller prematurely. Solving this open problem would probably require the development of new tools for directly bounding the revenue of interactive mechanisms - in sharp contrast to most optimal mechanisms in the literature which satisfy some version of the One-round Revelation Property.

Possibly, tools from dynamic mechanism design [4, 17] and from cheap talk [5] might be useful in dealing with this question, since both involve repeated interactions between parties before an outcome is effectively implemented. Nevertheless, our setting differs from them in fundamental ways: the reason interaction is necessary here is different from that in dynamic mechanism design. In our problem, information is just revealed by nature in the beginning of time. No exogenous signals are revealed during the interaction between seller and buyer. This fact brings our model closer to cheap talk, yet there is a substantial difference as in our setting talking is not "cheap" in the sense that payments are required so that the conversation continues.

2. Continuous Type Spaces and Structured Contexts: Our results fix a finite discrete type space and use tools from Linear and Convex Programming to design optimal mechanisms. Is it possible to obtain a general theory of "Selling Information" where the signals from nature come from arbitrary spaces? Esö and Szentes [11] are able to deal with real-valued signals but they severely restrict the utility function of the agents.

Is there a natural yet less restrictive condition on the context that makes the problem tractable for more generic spaces? Can one come up with a general condition that would be still be sufficient for the optimal mechanism to have explicit and natural representation (similar to Myerson's mechanism), rather than being a solution to some mathematical program?

3. Multiple buyers: In our work, we consider only two agents: a seller and a buyer. In the context of information advertisement, there are usually many sellers (many informationproviding agencies) and many buyers (advertisers). Those buyers do not simply solve a decision problem once they acquire the information, but play a game among each other.

A natural next step is to consider a variant of our model where there is a single seller but multiple buyers, who play a game after acquiring information. One needs to be careful when defining such problem since we must be sure that the game has an unique equilibrium or that for each possible outcome of the selling information phase, the Bayesian game played in the second phase has a focal equilibrium on which we can concentrate when reasoning about the first phase. Along those lines is the work of Esö and Szentes [12] and the recent paper by Kempe, Tardos and Syrgkanis [20].

4. Coupling Goods and Information: As mentioned earlier, there are many situations where goods and information are coupled together (for example, the restaurant example in the introduction). What is the correct model in which to analyze such situations? 
5. Dynamic selling of information: We analyzed a single interaction between the buyer and the seller. Alternatively one could consider an ongoing (possibly interactive) relation between those two parties, e.g. when the buyer is interested in information regarding multiple impressions. Can the seller benefit from this and extract strictly more revenue from the whole process than she would if running the optimal auction for each query individually? It is known that for traditional goods, selling bundles might generate strictly more revenue than selling goods individually. What is the form of the analogous results for information?

6. Computationally bounded agents: We assumed that the seller sends messages to the buyer coming from a certain distribution and the buyer uses Bayes' rule to learn about the state of the world from those messages. We do not impose computational constraints on the agents. If we were to assume computationally-constrained buyers, the seller could potentially take advantage of cryptographic primitives, for example sending an encrypted piece of information to the buyer and later selling the decryption key. How would such capabilities affect the results? Would the seller be able to exploit them in a profitable way?

\section{References}

[1] A. R. Admati and P. Pfleiderer. A monopolistic market for information. Journal of Economic Theory, 39(2):400-438, August 1986.

[2] A. R. Admati and P. Pfleiderer. Direct and indirect sale of information. Econometrica, 58(4):901-28, July 1990.

[3] S. Athey and J. Levin. The value of information in monotone decision problems. Working Papers 01003, Stanford University, Department of Economics, Feb. 2001.

[4] S. Athey and I. Segal. An efficient dynamic mechanism. Technical report, UCLA Department of Economics, Mar. 2007.

[5] R. J. Aumann and S. Hart. Long cheap talk. Econometrica, 71(6):1619-1660, November 2003.

[6] A. Ben-Tal and A. Nemirovskiu. Lectures on modern convex optimization: analysis, algorithms, and engineering applications. MPS-SIAM series on optimization. Society for Industrial and Applied Mathematics, 2001.

[7] D. Bergemann and J. Valimaki. Efficient dynamic auctions. Cowles Foundation Discussion Papers 1584, Cowles Foundation for Research in Economics, Yale University, Oct. 2006.

[8] S. Clifford. Your online clicks have value, for someone who has something to sell. New York Times, March 25, 2009, 2009.

[9] J. Cremer and R. P. McLean. Optimal selling strategies under uncertainty for a discriminating monopolist when demands are interdependent. Econometrica, 53(2):345-61, March 1985.

[10] J. Cremer and R. P. McLean. Full extraction of the surplus in bayesian and dominant strategy auctions. Econometrica, 56(6):1247-57, November 1988.

[11] P. Eső and B. Szentes. The price of advice. Discussion Papers 1416, Northwestern University, Center for Mathematical Studies in Economics and Management Science, Dec. 2004. 
[12] P. Eső and B. Szentes. Optimal information disclosure in auctions and the handicap auction. The Review of Economic Studies, 74(3):pp. 705-731, 2007.

[13] A. Gibbard. Manipulation of voting schemes: a general result. Econometrica, 41(4):587-601, 1973.

[14] J. Horner and A. Skrzypacz. Selling information. Cowles Foundation Discussion Papers 1743R, Cowles Foundation for Research in Economics, Yale University, Dec. 2009.

[15] P. R. Milgrom and R. J. Weber. A theory of auctions and competitive bidding. Econometrica, 50(5):1089-1122, September 1982.

[16] R. Myerson. Incentive-compatibility and the bargaining problem. Econometrica, 47:6173, 1979.

[17] A. Pavan, I. Segal, and J. Toikka. Dynamic mechanism design: Incentive compatibility, profit maximization and information disclosure. Carlo Alberto Notebooks 84, Collegio Carlo Alberto, 2008.

[18] N. Persico. Information acquisition in auctions. Econometrica, 68(1):135-148, January 2000.

[19] J. Robert. Continuity in auction design. Journal of Economic Theory, 55(1):169-179, October 1991.

[20] V. Syrgkanis, D. Kempe, and Éva Tardos. Selling third-party information for common-value auctions. Working Paper, 2012.

[21] H. Varian. Markets for information goods. IMES discussion paper series. Institute for Monetary and Economic Studies, Bank of Japan, 1999.

\section{A Proofs omitted from Section 2}

Proof of Theorem 2.6 : The idea of the proof is simple: we add in the root a buyer node where the buyer is asked to report his type $\theta$. For each branch, there is a copy of the original protocol where the seller simulates the behavior of the buyer.

Formally, we fix a context and a consider a protocol represented by a tree, distributions $\left\{\psi_{n}^{\omega}\right\}_{\omega \in \Omega}$ for each seller node $n$ and transfers $t(n)$ on each transfer node. Now, consider a set of moves for the buyer $\left\{\phi_{n}^{\theta}\right\}_{\theta \in \Theta}$ for each buyer and transfer node. We can assume that for any buyer node $n$, $\phi_{n}^{\theta}$ outputs $\perp$ with zero probability, since any other strategy is weakly dominated by a strategy in which the buyer does not defect at the buyer node and instead defect at the first transfer node encountered. Let $T$ represent this protocol.

Now, define the protocol $T_{\theta}$ as the same tree with all buyer nodes $n$ substituted by seller nodes where the seller moves according to $\phi_{n}^{\theta}$ for all $\omega \in \Omega$. Now, the tree $T_{\theta}$ consists only of transfer and seller nodes.

Now, design a new protocol with a buyer node in the root with $|\Theta|$ outgoing edges, one corresponding to each type. Attach to the $\theta$-branch a copy of $T_{\theta}$. Now, it is simple to see that an optimal committed (uncommitted) strategy is to report the true type and then follow the protocol (defecting in the nodes of $T_{\theta}$ corresponding to the nodes in $T$ where he defected in the original protocol). If any deviation is profitable now, the corresponding deviation would be profitable in the original protocol. Also, the revenue of the truthful strategy in the new protocol is clearly the same as the revenue of the original strategy in the original protocol. 


\section{B Proofs omitted from Section 3}

Proof of Theorem 3.1: Fix the context, consider a voluntary protocol and an optimal strategy $\phi$ for a committed buyer achieving revenue $R$. We show that we can reduce it to a protocol of the specified form, achieving the same revenue for uncommitted buyers. Let $Z(\omega, \theta)$ be the distribution over leaves obtained when we start from the root and use $\psi_{n}^{\omega}, \phi_{n}^{\theta}$ to move down the tree. Also, let $L$ be the set of leaves of the tree.

In the root of the reduced protocol, we place a buyer node with $|\Theta|$ edges out of it. This node corresponds to the buyer being asked to report his type. The children of the root are transfer nodes, each corresponding to a distinct type $\theta$. The amount at such a transfer node corresponds to the expected payment of type $\theta$ in the mechanism, i.e., $\mathbb{E}_{\omega}[\tau(Z(\omega, \theta))]$.

Now, consider the node $n$ who is the child of the transfer-node in the $\theta$-branch. Add a copy of $L$ as its children and for each $\omega$, have the seller use the distribution $Z(\omega, \theta)$.

Now, we need to check that the new protocol is indeed equivalent to the original one. Consider the uncommitted strategy where the buyers report their true types in the first node and then pay according to the transfer node. This strategy generates the same utility and payments as the ones in the original protocol, so one only needs to check that it is indeed an optimal strategy for the buyer.

It suffices to see that a buyer of type $\theta$ would not rather declare $\theta^{\prime}$ instead or defect in the middle of the reduced protocol. Defecting in the middle is clearly not beneficial, since the protocol is voluntary and the buyer does not learn anything until the last move. Also, if declaring $\theta^{\prime}$ were profitable for a buyer of type $\theta$, he would prefer to play according to $\phi^{\theta^{\prime}}$ in the original protocol. This happens for two reasons. First, it generates the same distribution over leaves given $\omega$ as declaring $\theta^{\prime}$ in the new protocol would. So the value of the deviations is the same. Second, it generates the same expected payment as declaring $\theta^{\prime}$ in the new protocol would. This depends crucially on independence. A buyer playing $\phi^{\theta^{\prime}}$ experiences the same expected payment regardless of his type, since $\omega$ is drawn from the same distribution (independent of $\theta$ ) and we thus visit the same nodes with the same probabilities in all cases.

Notice that the last step in the proof clearly does not hold if $\omega, \theta$ are correlated, since a buyer of type $\theta$ could change his declaration to $\theta^{\prime}$, but he can not prevent $\omega$ to be sampled with probability $\mu(\omega \mid \theta)$.

Proof of Observation 3.3: Simply notice that the utilities of each player for each contract in the original and new menu are the same.

Proof of Observation 3.4 : Let $q_{i} \in \Delta(\Omega)$ be the posterior associated with $i$, i.e. $q_{i}(\omega)=$ $\mathbb{P}(\omega \mid Y=i)$ and let $x\left(q_{i}\right)=\mathbb{P}(Y=i)$. Then:

$$
\sum_{q_{i} \in Q} x\left(q_{i}\right) \cdot q_{i}(\omega)=\sum_{i} \mathbb{P}(Y=i) \cdot \mathbb{P}(\omega \mid Y=i)=\mu(\omega)
$$

Conversely, given $(Q, x)$ satisfying $(F)$ we can define a variable $Y$ taking values in $[k]$ and set its joint distribution with $\omega$ to be $\mathbb{P}(\omega, Y=i)=x\left(q_{i}\right) \cdot q_{i}(\omega)$.

Proof of Lemma 3.5: The function $u$ defines piecewise linear functions $v_{\theta}: \Delta(\Omega) \rightarrow \mathbb{R}$. Each of them induces a partition of $\Delta(\Omega)$ in $|A|$ polytopes in which $v_{\theta}(\cdot)$ is linear. One can combine the $|\Theta|$ partitions, by taking the coarser partition of $\Delta(\Omega)$ that is simultaneously a subpartition of the one induced by $v_{\theta}$ for all $\theta$. This way we obtain a finite partition such that for all its regions all the $v_{\theta}$ functions are linear.

Now, let $Q^{*}$ be the set of vertices of the regions in this partition. Given any posterior $q \in \Delta(\Omega)$, if it is in region $R$ of the partition, one can write $q=\sum_{i} \gamma_{i} q_{i}$ where $q_{i} \in Q^{*}$ are vertices of region 
$R$ and $\sum_{i} \gamma_{i}=1, \gamma_{i} \geq 0$. Now, given a primal solution to $\mathbf{L P}_{\mathbf{1}}$, if $x_{\theta}(q)>0$ and $q \notin Q^{*}$, simply increase $x_{\theta}\left(q_{i}\right)$ by $\gamma_{i} x_{\theta}(q)$ and decrease $x_{\theta}(q)$ to zero. By the linearity of $v_{\theta}(\cdot), v_{\theta^{\prime}}(\cdot)$ in $R$, it is clear that the resulting solution is still feasible and has the same objective. By repeating this process as many times as needed, one ends with a solution where the support is in $Q^{*}$.

Proof of Lemma 3.6 : Given a convex programing problem $\min f(x)$ s.t. $A x \leq b$, in order to show that it can be solved exactly in polynomial time we need to show three things (see section 5.3 of [6]):

- that the function $f$ can be computed efficiently for each point $x_{0}$ in the domain.

- that for each point $x_{0}$ in the domain it is possible to calculate a subgradient $v \in \partial f\left(x_{0}\right)$. A subgradient is a vector $v \in \mathbb{R}^{n}$ such that $f(x)-f\left(x_{0}\right) \geq v^{t}\left(x-x_{0}\right)$

- that there is an optimal solution that can be expressed with polynomially-many bits.

Point 1 above is trivial. For point number 2 we use the linearity of the subgradient: if $v \in \partial g\left(x_{0}\right)$ and $w \in \partial h\left(x_{0}\right)$ then $v+w \in \partial(g+h)\left(x_{0}\right)$. And since the subgradient of linear functions is trivial, we now need to show how to compute the subgradient of $v_{\theta^{\prime}}(\cdot)$. Notice that $v_{\theta^{\prime}}(\cdot)$ is the maximum of linear functions. We use the fact that if $g(x)=\max _{j} g_{j}(x), g\left(x_{0}\right)=g_{i}\left(x_{0}\right)$ and $v_{i} \in \partial g_{i}\left(x_{0}\right)$, then $v_{i} \in \partial g\left(x_{0}\right)$, since:

$$
g(x)-g\left(x_{0}\right)=g(x)-g_{i}\left(x_{0}\right) \geq g_{i}(x)-g_{i}\left(x_{0}\right) \geq v_{i}^{t}\left(x-x_{0}\right)
$$

For point 3, Lemma 3.5 says that $\mathbf{L P}_{\mathbf{1}}$ can be written with finitely many variables. Therefore, the dual LP $\left(\mathbf{D L P}_{\mathbf{1}}\right)$ can be written with finitely many constraints. Now, the optimizer of the dual can be found by taking $O\left(|\Theta|^{2}\right)$ constraints, making them tight and solving the resulting linear system. This solution has polynomially many bits.

Given an optimal solution $q \in \Delta(\Omega)$, for each $\theta \in \Theta$ let $a(\theta)$ be a solution to $\max _{a} \mathbb{E}_{\omega \sim q} u(\omega, \theta, a)$. There is a polytope $\mathcal{P} \subseteq \Delta(\Omega)$ that consists of all posteriors $q^{\prime}$ such that actions $(a(\theta))_{\theta \in \Theta}$ remain optimal when the posterior is $q^{\prime}$. The objective function of the convex program is linear when restricted to $\mathcal{P}$, so the set of optimal solutions includes at least one extreme point of $\mathcal{P}$, and such an extreme point can be found in polynomial time. Recalling the construction of the set $Q^{*}$ in the proof of Lemma 3.5. we see that all of the extreme points of $\mathcal{P}$ are elements of $Q^{*}$, so we have established that our convex program has an optimal solution $q^{*}$ that belongs to $Q^{*}$, and that we can find such a $q^{*}$ in polynomial time, as claimed in the lemma.

The following example, discussed in Section 3, reveals that the Sealed Envelope Mechanism does not extract more than $\Omega\left(\frac{1}{|\Theta|}\right)$ of the revenue of the optimal mechanism.

Example B.1 Consider $\Omega=\{0,1\}, \Theta=[n]$ and $\mu(\omega, \theta)=\frac{2^{-\theta-1}}{1-2^{-n}}$. Now, for each $\theta$ we can represent $u(\theta, \omega, a)$ as a piecewise-linear function $v_{\theta}(\cdot)$ on $[0,1]$ where

$$
v_{\theta}(q)=\max _{a}[u(\theta, 1, a) \cdot q+u(\theta, 0, a) \cdot(1-q)]
$$

Let $v_{\theta}(\cdot)$ be the function interpolating the following three points: $(0,0),\left(1-\frac{1}{T^{\theta}}, 0\right),\left(1,2^{\theta}\right)$, where $T$ is some fixed large number. Now, the value of a buyer of type $\theta$ for the envelope is $2^{\theta}$ and occurs with probability $\mu(\theta)=O\left(2^{-\theta}\right)$, so any price $p$ will only be able to extract $O(1)$ revenue. 
Now, we show a Pricing Mappings Mechanism that can extract $\Omega(n)$ revenue. Consider the menu where the price of contract $\theta$ is $t_{\theta}=\left(1-\frac{1}{T}\right) 2^{\theta}-\epsilon$ and outputs the posterior $1-\frac{1}{T^{\theta+1}}$ w.p. $\frac{1}{2}$ and the posterior $\frac{1}{T^{\theta+1}}$ w.p. $\frac{1}{2}$. This is the same as outputting a variable $Y_{\theta}$ with joint distribution:

$$
\left[\mathbb{P}\left(Y_{\theta}, \omega\right)\right]=\left[\begin{array}{cc}
1 /\left(2 T^{\theta+1}\right) & 1 /\left(2\left(1-T^{\theta+1}\right)\right) \\
1 /\left(2\left(1-T^{\theta+1}\right)\right) & 1 /\left(2 T^{\theta+1}\right)
\end{array}\right]
$$

It is simple to check that this is a valid menu and that it generated revenue $\Omega\left(\left(1-\frac{1}{T}\right) n\right)$.

Proof of Theorem 3.7: $\quad$ Given any context, fix an optimal solution $\left\{x_{\theta}(q), t_{\theta}\right\}_{\theta}$ for $\mathbf{L P}_{\mathbf{1}}$. We can assume $\Theta=[n]$ and that the solution is such that $t_{1} \geq t_{2} \geq t_{3} \geq \ldots \geq t_{n}$. Let $Q_{\theta} \subset \Delta(\Omega)$ be the posteriors in the support of $x_{\theta}$. We can restrict our attention to $\mathbf{L P}_{\mathbf{1}}$ with variables $x_{\theta}(q)$ for $q \in \cup_{\theta} Q_{\theta}$.

For any given $\theta \in[n]$ we show that we can substitute $x_{\theta}(\cdot)$ by some $x_{\theta}^{\prime}(\cdot)$ with support at most $\theta+m-1$. In order to do so fix all $t_{\theta^{\prime}}$ and $x_{\theta^{\prime}}(\cdot)$ for all $\theta^{\prime} \neq \theta$. Now find $x_{\theta}^{\prime}(q), t_{\theta}^{\prime}$ solving the following system:

$$
\begin{aligned}
& \max t_{\theta}^{\prime} \text { s.t. } \\
& \qquad \sum_{q \in Q} v_{\theta}(q) x_{\theta}^{\prime}(q)-t_{\theta}^{\prime} \geq \max \left\{v_{\theta}(p), \max _{\theta^{\prime} \neq \theta} \sum_{q \in Q} v_{\theta}(q) x_{\theta^{\prime}}(q)-t_{\theta^{\prime}}\right\}, \quad\left(I R_{\theta}\right)+\left(I C_{\theta, \theta^{\prime}}, \theta^{\prime} \neq \theta\right) \\
& \sum_{q \in Q} v_{\theta^{\prime}}(q) x_{\theta^{\prime}}(q)-t_{\theta^{\prime}} \geq \sum_{q \in Q} v_{\theta}(q) x_{\theta}^{\prime}(q)-t_{\theta}, \quad \forall \theta^{\prime}<\theta, \quad\left(I C_{\theta^{\prime}, \theta}, \theta^{\prime}<\theta\right) \\
& \sum_{q \in Q} x_{\theta}^{\prime}(q) \cdot q(\omega)=\mu(\omega), \quad \forall \omega \in \Omega \\
& x_{\theta}^{\prime}(q) \geq 0, \quad \forall q \in Q
\end{aligned}
$$

The first constraint represents the $\operatorname{IR}_{\theta}$ combined with $I C_{\theta, \theta^{\prime}}$ for all $\theta^{\prime} \neq \theta$. Since all $x_{\theta^{\prime}}(\cdot), t_{\theta^{\prime}}$ are fixed, the rhs of the inequality is constant. The second family of constraints refer to the $\mathrm{IC}_{\theta^{\prime}, \theta}$ constraints for $\theta^{\prime}<\theta$.

The system of inequalities has $\theta+m$ constraints, so a basic feasible solution has support at most $\theta+m$, which corresponds to $t_{\theta}^{\prime}$ and $x_{\theta}^{\prime}(\cdot)$ such that $\left\|x_{\theta}^{\prime}(\cdot)\right\|_{0} \leq \theta+m-1$. Consider the solution we get from substituting $x_{\theta}(\cdot)$ by $x_{\theta}^{\prime}(\cdot)$. The only constraints in $\mathbf{L} \mathbf{P}_{\mathbf{1}}$ which can be violated are:

$$
\sum_{q} v_{\theta^{\prime}}(q) x_{\theta^{\prime}}(q)-t_{\theta^{\prime}} \geq \sum_{q} v_{\theta}(q) x_{\theta}(q)-t_{\theta}
$$

for some $\theta^{\prime}>\theta$. If this happens for some $\theta^{\prime}$, substitute $\left(x_{\theta^{\prime}}, t_{\theta^{\prime}}\right)$ by $\left(x_{\theta}^{\prime}, t_{\theta}\right)$. It is simple to see that the solution obtained is feasible and that the revenue did not decrease.

Finally, observe that the theorem follows as

$$
\sum_{\theta}\left\|x_{\theta}\right\|_{0} \leq \sum_{\theta}(m-1+\theta)=m n+\left(\begin{array}{l}
n \\
2
\end{array}\right)
$$

We show in Example B.2 that the quadratic dependence in $|\Theta|$ is necessary if one wants to obtain the optimal revenue. 
Example B.2 Consider $\Omega=\{0,1\}, \Theta=[n]$ and as in Example B.1 represent $u$ by piecewise-linear convex functions $v_{\theta}:[0,1] \rightarrow \mathbb{R}$. For some small enough parameter $\delta>0$, define $v_{\theta}$ as the function that interpolates points

$$
(0,0),(1-\delta \theta, 0),\left(1-\delta(\theta-1), \delta^{2 \theta-2}\right),\left(1-\delta(\theta-2), \delta^{2 \theta-1}\right), \ldots,\left(1-\delta, \delta^{\theta}\right),\left(1, \delta^{\theta-1}\right)
$$

For example, $v_{1}$ is the function interpolating $(0,0),(1-\delta, 0),(1,1)$. Function $v_{2}$ interpolates $(0,0),(1-$ $2 \delta, 0),\left(1-\delta, \delta^{2}\right),(1, \delta)$ and so on.

For this example we represent all the posteriors $q \in \Delta(\Omega)$ by a real number $q(1)$, the posterior probability that $\omega=1$.

Now, define $\mu$ such that $\mu(\omega=0)=\mu(\omega=1)=\frac{1}{2}$. Before we define the distribution over $\Theta$ we note two things about the primal LP:

1. there is an optimal solution to $\mathbf{L P}_{\mathbf{1}}$ where all posteriors are in $Q=\{0,1-n \delta, 1-(n-$ 1) $\delta, \ldots, 1-\delta, 1\}$ by the argument in the proof of the Interesting Posteriors Lemma (Lemma 3.5).

2. we can write the primal only with the variables $x_{\theta}(q)$ for $q \in Q$. This generates a finite $L P$. Further notice that the polytope that defines the feasible set is define by $v_{\theta}(\cdot)$ and the distribution $\mu(\omega)$, both already defined. The distribution $\mu(\theta)$ just appears in the objective function.

Since the feasible region is already defined and it is a polytope (since by restricting to $Q$ we have a finite number of constraints), let $V$ be the set of all vertices of this polytope. Define $\tilde{V} \subset \mathbb{R}_{+}$as the set of all $t$ such that there is $\left(x_{\theta^{\prime}}, t_{\theta^{\prime}}\right)_{\theta^{\prime} \in \Theta} \in V$ and $\theta$ such that $t=t_{\theta}$. In other words, it is the set of all prices appearing in the vertices of the feasible regions. Given that, pick some $\epsilon$ such that:

$$
\epsilon<\frac{1}{n} \cdot \frac{\min _{t, t^{\prime} \in \tilde{V}, t \neq t^{\prime}}\left|t-t^{\prime}\right|}{\max _{t, t^{\prime} \in \tilde{V}}\left|t-t^{\prime}\right|}
$$

and define $\mu(\theta)=\epsilon^{\theta} \cdot \frac{1-\epsilon}{\epsilon\left(1-\epsilon^{n}\right)}$. Now the context is define, we can analyze the optimal menu of contracts for this context. We will show that the optimal menu is unique and $\sum_{\theta}\left\|x_{\theta}\right\|_{0}=\Theta\left(n^{2}\right)$.

We claim that for this value of $\epsilon$, the optimal solution to the primal LP can be found by optimizing $t_{1}$ over the feasible set, fixing it, then optimizing for $t_{2}$ (having $x_{1}, t_{1}$ fixed), and so on. The definition of $\epsilon$ guarantees that no possible gain in $t_{\theta}+1, \ldots, t_{n}$ can justify a decrease in $t_{\theta}$.

For example, for $\theta=1$, if we want to find $\max t_{1}$ with respect to the feasible set of the primal $L P$, the unique solution is to take $t_{1}$ to be the surplus $\frac{1}{2}\left[v_{1}(0)+v_{1}(1)\right]-v_{1}\left(\frac{1}{2}\right)=\frac{1}{2} v_{1}(1)$, since $v_{\theta}(0)=v_{\theta}\left(\frac{1}{2}\right)=0$ by construction, and $x_{1}(0)=x_{1}(1)=\frac{1}{2}$. Notice that buyer of type 1 gets no utility from this contract (the seller is able to extract full surplus). Now, after fixing $\left(x_{1}, t_{1}\right)$ we need to guarantee that for all the other contracts buyer with $\theta=1$ is not able to extract any utility from them.

We claim that there is an unique optimal solution and the support of $x_{\theta}$ is $0,1-(\theta-1) \delta, 1-$ $(\theta-2) \delta, \ldots, 1$. Moreover, in the optimal solution, the utility a buyer of type $\theta$ extracts from his own contract is zero, i.e., $\sum_{q} x_{\theta}(q) v_{\theta}(q)-t_{\theta}=v_{\theta}(p)$. We show this by induction on $\theta$ : in order to find the optimal $\left(x_{\theta}, t_{\theta}\right)$ one needs to solve the following program (we are using the fact that the players $\theta^{\prime}<\theta$ get zero utility from their own contracts): 


$$
\begin{aligned}
& \max t_{\theta} \text { s.t. } \\
& \sum_{q \in Q} v_{\theta}(q) x_{\theta}(q)-t_{\theta} \geq 0,, \quad\left(I R_{\theta}\right) \\
& \sum_{q \in Q} v_{\theta^{\prime}}(q) x_{\theta}(q)-t_{\theta} \leq 0, \quad \forall \theta^{\prime}<\theta, \quad\left(I C_{\theta^{\prime}}, \theta^{\prime}<\theta\right) \\
& \sum_{q \in Q}(q, 1-q) \cdot x_{\theta}(q)=(1 / 2,1 / 2), \quad\left(F_{\theta}\right) \\
& x_{\theta}(q) \geq 0,
\end{aligned}
$$

Substituting the values of $v_{\theta}(\cdot)$, we get:

$$
\begin{aligned}
& \sum_{j=0}^{\theta-1} \delta^{\theta+j-1} x_{\theta}(1-j \delta) \geq t_{\theta} \\
& \sum_{j=0}^{\theta^{\prime}-1} \delta^{\theta^{\prime}+j-1} x_{\theta}(1-j \delta) \leq t_{\theta}, \forall \theta^{\prime}=1, \ldots, \theta-1
\end{aligned}
$$

Now we claim that in the solution maximizing $t_{\theta}$ all the inequalities above must be tight. Since it is a triangular system, it guarantee that the support of $x_{\theta}$ needs to be of size $\theta+1$.

Consider an optimal $\left(x_{\theta}, t_{\theta}\right)$ maximizing $t_{\theta}$ and suppose that one of the constraints above is not tight. Clearly if the IR constraint is not tight, we can simply increase $t_{\theta}$, which is an absurd since the solution is optimal.

Now, suppose that one of the IC constraints are not tight. Then let $\theta^{\prime}$ be the largest $\theta^{\prime}$ such that $I C_{\theta^{\prime}}$ is not tight. Now, it must be the case that $x_{\theta}\left(1-\theta^{\prime} \delta\right)>0$. In order to see that, consider two cases (i) $\theta^{\prime}<\theta-1$ : this would imply that constraint $I C_{\theta^{\prime}+1}$ is also slack, violating the maximality of $\theta^{\prime}$ and (ii) $\theta^{\prime}=\theta-1$ : this would cause the solution to be infeasible due to a conflict between $I C_{\theta-1}$ and $I R$.

Given that, one can construct a new solution by decreasing $x_{\theta}\left(1-\theta^{\prime} \delta\right)$ by a small amount $\rho$ and increasing $x_{\theta}\left(1-\left(\theta^{\prime}-1\right) \delta\right)$ by the amount $\delta \rho$. This keeps IR and IC constraints still valid,

but violates $\sum_{q \in Q}(q, 1-q) \cdot x_{\theta}(q)=(1 / 2,1 / 2)$. It is simple to see one can increase $x_{\theta}(0)$ and $x_{\theta}(1-(\theta-1) \delta)$ by suitable values to restore this constraint. By doing so, we do not violate any of the IC constraints and we make the IR constraint a little bit more slack, which allows us to increase $t_{\theta}$. Since it was optimal from beginning, then all IC constraints must be tight.

Now, since all IC constraints and IR constraints are tight, and together they form a triangular system, it is easy to see the solution must have support $\theta+1$. Now, summing for all $\theta$, we get $\sum_{\theta}\left\|x_{\theta}\right\|_{0}=\sum_{\theta} 1+\theta$ exactly matching the bound in theorem 3.7 for $m=2$.

\section{Proofs omitted from Section 4.1}

Proof : [of Theorem 4.1] Using the same notation of Theorem 3.1, we can mimic that proof, but whenever constructing the new protocol, we add a seller node just after the buyer node with a transition to a copy of $L$ using $Z(\omega, \theta)$ in the $\theta$-branch. Now, for each child of the seller node, if it corresponds to a leaf $\ell$ in the original protocol, add a transfer node with amount $\tau(\ell)$.

Again, the buyer committed strategy where each buyer reports his type truthfully generates the same outcomes and payments as the original protocol. We only need to argue that it is optimal. The argument is very similar to the one in Theorem 3.1. however the inversion of the buyer and 
seller nodes allows us to prove point 2 without independence. We notice that if a buyer of type $\theta$ plays using $\theta^{\prime}$ he has the same distribution over leaves as he would get if he played $\phi^{\theta^{\prime}}$ in the original protocol - by the new construction, it also means the same distribution over transfers.

Computing the optimal Pricing Outcomes Mechanism. Consider a buyer of type $\theta$ that buys a contract $(Y, t)$. Let $Y$ be represented by a family $\psi^{\omega} \in \Delta(\mathcal{S})$ for each $\omega \in \Omega$. If a signal $s \in \mathcal{S}$ is observed by a buyer with type $\theta$ and also by an outside observer, the two observers have different beliefs on $\omega$ - the buyer's belief is $\mu(\cdot \mid \theta)$ while the outside observer's is $\mu(\cdot)$ — so Bayes' rule dictates their respective posteriors as follows.

$$
q_{\theta}^{s}(\omega)=\mathbb{P}(\omega \mid \theta, s)=\frac{\mathbb{P}(\omega, s, \theta)}{\mathbb{P}(s, \theta)}=\frac{\mathbb{P}(\omega, s) \cdot \mu(\theta \mid \omega)}{\mathbb{P}(s) \cdot \mathbb{P}(\theta \mid s)}, \quad q^{s}(\omega)=\mathbb{P}(\omega \mid s)=\frac{\mathbb{P}(\omega, s)}{\mathbb{P}(s)}
$$

So, up to rescaling there is a very simple linear transformation that relates the posteriors of the buyer of type $\theta$ for a signal and the posterior of the outside observer. Let $D_{\theta}$ be an $|\Omega| \times|\Omega|$ diagonal matrix with $\mu(\theta \mid \omega)$ in the diagonal. Then, we can write:

$$
q_{\theta}^{s}=\frac{D_{\theta} \cdot q^{s}}{\mathbb{P}(\theta \mid s)}
$$

Given the 1-1 mapping between posteriors from the perspective of buyer of type $\theta$ and posteriors with respect to an outside observer, from this point on, whenever referring to posteriors, we always refer to posteriors with respect to the outside observer and apply the suitable transformation when necessary.

Representation of contracts. Analogously to what was done in the independent case, we represent $Y$ by a distribution over (outside observer) posteriors. We can use Observation 3.3 to see that it is possible to assume w.l.o.g. that the posteriors associated with each $s \in \mathcal{S}$ are different. So, we can represent $Y_{\theta}$ as a distribution over the set of posteriors $\Delta(\Omega)$. More formally, we represent $Y$ by $x: \Delta(\Omega) \rightarrow \mathbb{R}_{+}$with finite support satisfying the feasibility condition in Observation 3.4. Similarly $t$ can be represented as $t: \Delta(\Omega) \rightarrow \mathbb{R}$, simply by associating each signal with its posterior. Given that, we can represent the valuation that a buyer of type $\theta$ has for contract $(Y, t)$ as:

$$
\begin{aligned}
\mathbb{E}_{Y} & {\left[\max _{a} \mathbb{E}[u(\omega, \theta, a) \mid \theta, Y]\right]=\sum_{s \in \mathcal{S}} \mathbb{P}(Y=s \mid \theta) \cdot v_{\theta}\left(\frac{D_{\theta} q^{s}}{\mathbb{P}(\theta \mid s)}\right)=\sum_{s \in \mathcal{S}} \frac{\mathbb{P}(s \mid \theta)}{\mathbb{P}(\theta \mid s)} \cdot v_{\theta}\left(D_{\theta} q^{s}\right)=} \\
& =\sum_{s \in \mathcal{S}} \frac{\mathbb{P}(s)}{\mathbb{P}(\theta)} v_{\theta}\left(D_{\theta} q^{s}\right)=\frac{1}{\mathbb{P}(\theta)} \cdot \sum_{q} v_{\theta}\left(D_{\theta} q\right) \cdot x(q)
\end{aligned}
$$

where the first line uses the fact that $v_{\theta}$ is homogeneous of degree 1 , and the transition from the first to the second line makes use of Bayes' rule in the form $\mathbb{P}(s \mid \theta) / \mathbb{P}(\theta \mid s)=\mathbb{P}(s) / \mathbb{P}(\theta)$. The buyer's expected payment for this contract is given by:

$$
\begin{aligned}
\mathbb{E}_{Y} & {[t(Y=s) \mid \theta]=\sum_{s \in \mathcal{S}} t(s) \cdot \frac{\sum_{\omega} \mathbb{P}(s, \omega, \theta)}{\mathbb{P}(\theta)}=\frac{1}{\mathbb{P}(\theta)} \sum_{s \in \mathcal{S}} t(s) x\left(q^{s}\right) \sum_{\omega} q^{s}(\omega) \mu(\theta \mid \omega)=} \\
& =\frac{1}{\mathbb{P}(\theta)} \sum_{q} t(q) x(q) \cdot\left(\mathbb{1}^{t} D_{\theta} q\right)
\end{aligned}
$$

where $\mathbb{1}$ is the vector in $\mathbb{R}^{\Omega}$ with all 1 coordinates. 
Linear Programming Formulation: Now, in order to optimize the revenue from the protocol we need to design a menu of contracts that is valid, in the sense that player $\theta$ indeed chooses contract $\left(Y_{\theta}, t_{\theta}\right)$ and this choice is voluntary. One can define this in a similar manner to Definition 3.2 making the appropriate changes. Since the definition and follow up discussion very closely mirrors the one in the independent case, we proceed to designing the LP. As in the independent case, there is an "interesting posteriors lemma" that allows us to limit our attention to contracts whose posterior vectors belong to a predefined finite set $Q^{*}$. This time the set $Q^{*}$ depends not only on $u$ but on $\mu$, since the operator $D_{\theta}$ depends on $\mu$.

Lemma C.1 (Interesting Posteriors for correlated signals) Given $u: \Theta \times \Omega \times A \rightarrow \mathbb{R}$ and $\mu \in \Delta(\Theta \times \Omega)$, there is a finite set $Q^{*} \subset \Delta(\Omega)$ such that for all $\mu$, the maximum revenue that can be extracted by any protocol can also be extracted by a protocol that is limited to use posteriors in $Q^{*}$. Moreover, all the elements $q \in Q^{*}$ can be represented with polynomially many bits.

Proof : [sketch] The proof exactly parallels the proof of Lemma 3.5, except that now we must use the linear transformation $D_{\theta}$ to translate every buyer's piecewise-linear valuation function $v_{\theta}$ into the outside observer's frame of reference. In other words, we partition $\Delta(\Omega)$ into polyhedral pieces on which each of the function $q \mapsto v_{\theta}\left(D_{\theta} q\right)$ is linear, and let $Q^{*}$ be the set of vertices of the regions in this partition.

Let us continue deriving the LP formulation of the revenue maximization problem. The variables in the $\mathrm{LP}$ are $\left(x_{\theta}(q), \tilde{t}_{\theta}(q)\right)$ for all $\theta \in \Theta, q \in Q^{*}$. We define $\tilde{t}_{\theta}(q)=t_{\theta}(q) \cdot x_{\theta}(q)$. We write a linear program that expresses maximization of revenue subject to the analogues of the usual IR, IC and feasibility constraints for the correlated case.

$$
\begin{array}{lr}
\mathbf{L P}_{\mathbf{2}}: \max \sum_{\theta} \sum_{q}\left(\mathbb{1}^{t} D_{\theta} q\right) \cdot \tilde{t}_{\theta} \text { s.t. } & \\
\sum_{q} v_{\theta}\left(D_{\theta} q\right) x_{\theta}(q)-\left(\mathbb{1}^{t} D_{\theta} q\right) \tilde{t}_{\theta}(q) \geq v_{\theta}\left(D_{\theta} p\right), & \forall \theta, \quad\left(I R_{\theta}\right) \\
\sum_{q} v_{\theta}\left(D_{\theta} q\right) x_{\theta}(q)-\left(\mathbb{1}^{t} D_{\theta} q\right) \tilde{t}_{\theta}(q) \geq \sum_{q} v_{\theta}\left(D_{\theta} q\right) x_{\theta^{\prime}}(q)-\left(\mathbb{1}^{t} D_{\theta} q\right) \tilde{t}_{\theta^{\prime}}(q), & \forall \theta^{\prime} \neq \theta, \quad\left(I C_{\theta, \theta^{\prime}}\right) \\
\sum_{q} x_{\theta}(q) \cdot q(\omega)=\mu(\omega), & \forall \theta, \omega, \quad\left(F_{\theta}\right) \\
x_{\theta}(q) \geq 0, & \forall q, \theta
\end{array}
$$

Remark C.2 Before solving $\mathbf{L P}_{\mathbf{2}}$ to find the optimal mechanism, it is important to remark that the variables of the LP correspond to the posteriors as seen an outside observer, i.e., someone that knows $\mu$ but only observes the signal that the seller outputs and not the value of $\theta$. Equation $(B U)$ shows how to calculate the posteriors of the buyer given the posterior of the outside observer. It is instructive to consider the feasibility constraint $\left(F_{\theta}\right)$ in $\mathbf{L P}_{\mathbf{2}}$ when viewed from the perspective of the buyer. First, we can re-write $\left(F_{\theta}\right)$ as: $\sum_{s} \mathbb{P}(s) \cdot q^{s}(\omega)=\mu(\omega)$, multiplying by $\mu(\theta \mid \omega)$ and substituting by the definition of $q_{\theta}^{s}$ given by the Bayesian update equation $(B U)$ we have:

$$
\sum_{s} \mathbb{P}(s) \cdot q_{\theta}^{s}(\omega) \cdot \mathbb{P}(\theta \mid s)=\mu(\omega, \theta)
$$

Now, by manipulating the probabilities, we get:

$$
\sum_{s} \mathbb{P}(s \mid \theta) \cdot q_{\theta}^{s}(\omega)=\mu(\omega \mid \theta)
$$


which shows that the feasibility constraints from the perspective of the buyers is simply the feasibility constraints from the perspective of the observer transformed by the Bayesian update $(B U)$ operator.

Solving $\mathbf{L P}_{\mathbf{2}}$ can be done using exactly the same techniques used in the independent case: passing to the dual and using convex programming to design a separation oracle. We omit the details on how to solve it, since it essentially mirrors the independent case.

Once we have a solution $x_{\theta}(q), \tilde{t}_{\theta}(q)$ with support on $\hat{Q} \subseteq Q^{*}$, we want to recover a solution $\left(x_{\theta}, t_{\theta}\right)$. Clearly one can obtain $t_{\theta}(q)$ by taking $\tilde{t}_{\theta}(q) / x_{\theta}(q)$ whenever $x_{\theta}(q)$ is positive. What if it is equal to zero? In order to solve this problem we take the following steps: (1) we show that there is one feasible solution with revenue at least $(1-\epsilon)$ of the optimal, where all the IC and IR constraints are slack (2) then we increase $x_{\theta}(q)$ slightly for all $q \in \hat{Q}$ and renormalize so that feasibility holds and (3) we safely calculate $t_{\theta}(q)=\tilde{t}_{\theta}(q) / x_{\theta}(q)$, since now we are sure that $x_{\theta}(q)$ is positive. The following lemmas show how to perform steps (1) and (2):

Lemma C.3 (Strict Preferences) Given an optimal solution $\left(x_{\theta}, \tilde{t}_{\theta}\right)$ to $\mathbf{L P}_{\mathbf{2}}$, then there is a solution $\left(x_{\theta}^{\prime}, \tilde{t}_{\theta}^{\prime}\right)$ whose objective is at least a $(1-\epsilon)$-fraction of the optimal, such that all IC and IR constraints are slack, except possibly $I C_{\theta, \theta^{\prime}}$ when $\left(x_{\theta}^{\prime}, \tilde{t}_{\theta}^{\prime}\right)=\left(x_{\theta^{\prime}}^{\prime}, \tilde{t}_{\theta^{\prime}}^{\prime}\right)$, i.e., when the contracts for $\theta$ and $\theta^{\prime}$ are exactly the same.

Proof : First, suppose a certain IC constraint is tight, say:

$$
\sum_{q} v_{\theta}\left(D_{\theta} q\right) x_{\theta}(q)-\left(\mathbb{1}^{t} D_{\theta} q\right) \tilde{t}_{\theta}(q)=\sum_{q} v_{\theta}\left(D_{\theta} q\right) x_{\theta^{\prime}}(q)-\left(\mathbb{1}^{t} D_{\theta} q\right) \tilde{t}_{\theta^{\prime}}(q) .
$$

Then it must be the case that $\left(\mathbb{1}^{t} D_{\theta} q\right) \tilde{t}_{\theta}(q) \geq\left(\mathbb{1}^{t} D_{\theta} q\right) \tilde{t}_{\theta^{\prime}}(q)$. Otherwise one could replace $\left(x_{\theta}, \tilde{t}_{\theta}\right)$ with $\left(x_{\theta^{\prime}}, \tilde{t}_{\theta^{\prime}}\right)$ as buyer $\theta$ 's contract and improve revenue while still getting a feasible solution. We can assume w.l.o.g. that either $\left(\mathbb{1}^{t} D_{\theta} q\right) \tilde{t}_{\theta}(q)>\left(\mathbb{1}^{t} D_{\theta} q\right) \tilde{t}_{\theta^{\prime}}(q)$ or $\left(x_{\theta}, \tilde{t}_{\theta}\right)=\left(x_{\theta^{\prime}}, \tilde{t}_{\theta^{\prime}}\right)$. Otherwise, if $\left(\mathbb{1}^{t} D_{\theta} q\right) \tilde{t}_{\theta}(q)=\left(\mathbb{1}^{t} D_{\theta} q\right) \tilde{t}_{\theta^{\prime}}(q)$, one can simply replace $\left(x_{\theta}, \tilde{t}_{\theta}\right)$ with $\left(x_{\theta^{\prime}}, \tilde{t}_{\theta^{\prime}}\right)$.

Now, consider the menu where the contract for a buyer of type $\theta$ is $\left(x_{\theta}^{\prime}, \tilde{t}_{\theta}^{\prime}\right)=\left(x_{\theta},(1-\epsilon) t_{\theta}\right)$. We claim that for sufficiently small $\epsilon$, this is feasible and all constraints are slack.

Any constraint that held strictly is still strict for sufficiently small $\epsilon$. Any tight IR constraint becomes clearly strict for any $\epsilon>0$; in addition, no IR constraint becomes violated. Now for the IC constraints that were previously tight and $\left(x_{\theta}, \tilde{t}_{\theta}\right) \neq\left(x_{\theta^{\prime}}, \tilde{t}_{\theta^{\prime}}\right)$, we know that $\left(\mathbb{1}^{t} D_{\theta} q\right) \tilde{t}_{\theta}(q)>$ $\left(\mathbb{1}^{t} D_{\theta} q\right) \tilde{t}_{\theta^{\prime}}(q)$, hence the inequality becomes strict.

Lemma C.4 Given an optimal solution $\left(x_{\theta}, \tilde{t}_{\theta}\right)$ to $\mathbf{L P}_{\mathbf{2}}$ where all the $I C$ and $I R$ constraints are slack except possibly $I C_{\theta, \theta^{\prime}}$ when $\left(x_{\theta}, \tilde{t}_{\theta}\right)=\left(x_{\theta^{\prime}}, \tilde{t}_{\theta^{\prime}}\right)$, there is another solution $\left(x_{\theta}, \tilde{t}_{\theta}^{\prime}\right)$ with the same objective such that $x_{\theta}(q)>0$ for all $q \in \hat{Q}$.

Proof : Recall that $p \in \mathbb{R}_{+}^{\Omega}$ is the prior on $\omega$, i.e., $p(\omega)=\mu(\omega)$. We can assume wlog that $p(\omega)>0, \forall \omega \in \Omega$. (Otherwise, we can simply restrict our attention to a subset of $\Omega$ ). Therefore, $p$ is in the relative interior of $\Delta(\Omega)$. For all $q \in \Delta(\Omega)$ there is $r(q) \in \Delta(\Omega)$ and $\gamma(q) \in[0,1]$ such that:

$$
p=\gamma(q) \cdot q+(1-\gamma(q)) \cdot r(q)
$$

Now, consider $\tilde{t}_{\theta}^{\prime}=\tilde{t}_{\theta}$ for all $\theta$ and $x_{\theta}^{\prime}(q)=(1-\delta) x_{\theta}(q)$. Now, for all $q \in \hat{Q}$ increase $x_{\theta}^{\prime}(q)$ by $\frac{1}{|\hat{Q}|} \gamma(q) \delta$ and $x_{\theta}^{\prime}(r(q))$ by $\frac{1}{|\hat{Q}|}(1-\gamma(q)) \delta$. If $\delta$ is sufficiently small, the IC and IR constraints are not violated. For the IC constraints that held with equality and $\left(x_{\theta}, \tilde{t}_{\theta}\right)=\left(x_{\theta^{\prime}}, \tilde{t}_{\theta^{\prime}}\right)$, they still hold since 
$\left(x_{\theta}^{\prime}, \tilde{t}_{\theta}^{\prime}\right)=\left(x_{\theta^{\prime}}^{\prime}, \tilde{t}_{\theta^{\prime}}^{\prime}\right)$. It is trivial to check that by construction, the feasibility constraints still hold.

Also, we would like to observe that as observed in Theorem 3.7 for the Pricing Mappings Mechanism, the optimal protocol in this case can be represented by a tree of $O\left(n^{2}+m n\right)$ size. Given the structural similarities between $\mathbf{L P}_{\mathbf{1}}$ and $\mathbf{L P}_{\mathbf{2}}$, the proof follows by a simple adaptation of the proof of Theorem 3.7. Formally:

Observation C.5 Let $n=|\Theta|$ and $m=|\Omega|$. The program $\mathbf{L P}_{\mathbf{2}}$ has a solution where $\left\|x_{\theta}\right\|_{0} \leq$ $O(m+n)$ for all $\theta$.

\section{Proofs omitted from Section 4.2}

This appendix contains the full details of the material sketched in Section 4.2

\section{D.1 Semicontinuity Proofs}

Theorem D.1 The revenue function $R: \mathcal{C} \rightarrow \mathbb{R}$ is lower-semicontinuos.

Proof : Consider a sequence of contexts $\left(u_{i}, \mu_{i}\right)$ such that $\left(u_{i}, \mu_{i}\right) \rightarrow(u, \mu)$, then consider a revenue optimal mechanism for the context $(u, \mu)$. Invoking Lemma C.3 we know that for every $\epsilon>0$, there is a mechanism for context $(u, \mu)$ generating revenue at least $(1-\epsilon) \cdot R(u, \mu)$ such that all IR constraints are slack and all IC constraints for non-identical-contracts are slack. Given that the inequalities are strict and that the constraints are continuous in the context, then for sufficiently large $i$, the mechanism is feasible for $\left(u_{i}, \mu_{i}\right)$, so: $\lim \inf R\left(u_{i}, \mu_{i}\right) \geq R(u, \mu)-\epsilon$ for all $\epsilon>0$.

We note that a proof in the same spirit for the Cremer and McLean setting can be found in Robert [19]. Notice that the heart of the proof is the application of Lemma C.3. In this specific ingredient, our proof is different then the one of Robert.

The proof of Theorem D.1 can be interpreted as a robustness result: if the seller believes that the context $(u, \mu)$ and designs an protocol extracting $R(u, \mu)$, but the real context is actually slightly different $\left(u^{\prime}, \mu^{\prime}\right)$, the seller can design a protocol extracting revenue $(1-\epsilon) \cdot R(u, \mu)$ from any context $\left(u^{\prime}, \mu^{\prime}\right)$ that is sufficiently close to $(u, \mu)$.

This does not mean, however, that the seller is close to the optimal revenue, since it might be the case that the a context $\left(u^{\prime}, \mu^{\prime}\right)$ allows the seller to extract a lot more revenue then $(u, \mu)$ even though the two contexts are close.

Two Continuous Mechanisms. In what follows we present two mechanisms that fix the two highlighted problems of the Pricing Outcomes Mechanism: they are continuous and they do not involve transfers from the seller to the buyers. Then we compare their revenue-extration power with the optimal mechanism. For independent $(\omega, \theta)$, they extract optimal revenue, but there are contexts with correlated $(\omega, \theta)$ where they extract only a $\Omega\left(|\Theta|^{-1}\right)$-fraction of the optimal revenue.

1. Pricing Outcomes with No Positive Transfers: The auctioneer offers to the buyers a menu of contracts $\left\{\left(Y_{\theta}, t_{\theta}\right)\right\}_{\theta}$, where $Y_{\theta}$ is a random variable taking values in $\mathcal{S}_{\theta}$ and $t_{\theta}: \mathcal{S} \rightarrow \mathbb{R}_{+}$. The only change with respect to the optimal mechanism is that we require $t_{\theta}(s) \geq 0, \forall s \in \mathcal{S}_{\theta}$. To find the optimal mechanism of this type, one can simply solve $\mathbf{L P}_{\mathbf{2}}$ with the additional constraints that $\tilde{t}_{\theta}(q) \geq 0, \forall \theta, q$. 
2. Pricing Mappings: The auctioneer offers to the buyers a menu of contracts $\left\{\left(Y_{\theta}, t_{\theta}\right)\right\}_{\theta}$ where $Y_{\theta}$ is a random variable taking values in $\mathcal{S}_{\theta}$ and $t_{\theta} \in \mathbb{R}_{+}$is a fixed value that the buyer needs to pay in order to get the signal $Y_{\theta}$. The optimal mechanism of this type can be found using a simplified version of $\mathbf{L P}_{\mathbf{2}}$ : simply substitute all the occurrences of $\sum_{q}\left(\mathbb{1}^{t} D_{\theta} q\right) \tilde{t}_{\theta^{\prime}}(q)$ by $\mu(\theta) \cdot t_{\theta^{\prime}}$.

We presented four types of mechanisms which are increasing in complexity and revenue-extracting power. The following diagram expresses which types are a special case of which:

$$
\left\{\begin{array}{c}
\text { Sealed } \\
\text { Envelope }
\end{array}\right\} \subseteq\left\{\begin{array}{c}
\text { Pricing } \\
\text { Mappings }
\end{array}\right\} \subseteq\left\{\begin{array}{c}
\text { Pricing Outcomes } \\
t \geq 0
\end{array}\right\} \subseteq\left\{\begin{array}{c}
\text { Pricing } \\
\text { Outcomes }
\end{array}\right\}
$$

For a given context $(u, \mu) \in \mathcal{C}$, we define $R_{e}(u, \mu), R_{c}(u, \mu)$ and $R_{p}(u, \mu)$ as the optimal revenue that can be obtained using a Sealed Envelope Mechanism, Pricing Mappings Mechanism and Pricing Outcomes With No Positive Transfers Mechanism, respectively. We know that:

$$
R_{e}(u, \mu) \leq R_{c}(u, \mu) \leq R_{p}(u, \mu) \leq R(u, \mu)
$$

And by Theorem 3.1 we know that for independent $\mu$, i.e, $\mu(\omega, \theta)=\mu(\omega) \cdot \mu(\theta)$, we have:

$$
R_{e}(u, \mu) \leq R_{c}(u, \mu)=R_{p}(u, \mu)=R(u, \mu)
$$

First we will show that $R_{p}, R_{c}$ and $R_{e}$ are continuous in $\mathcal{C}$ and then we analyze the gap in their revenue extraction power. By a similar argument then the one in Theorem D.1, it is simple to see that they are lower-semicontinuous. In order to prove continuity, we only need to show uppersemicontinuity. We say that a function $f: \mathbb{R}^{k} \rightarrow \mathbb{R}$ is upper-semicontinuous if for for all converging sequences $x_{i} \rightarrow x, f(x) \geq \limsup f\left(x_{i}\right)$. A function is continuous if it is both lower-semicontinuous and upper-semicontinuous.

Lemma D.2 The revenue function $R_{c}: \mathcal{C} \rightarrow \mathbb{R}$ is upper-semicontinuous.

Proof : Consider a context $(u, \mu) \in \mathcal{C}$ and $\left(u_{i}, \mu_{i}\right) \rightarrow(u, \mu)$. Passing to a subsequence if necessary we can assume that $R_{c}\left(u_{i}, \mu_{i}\right)$ converges to the limsup of the original sequence. Now, we need to prove that $R_{c}(u, \mu) \geq \lim R_{c}\left(u_{i}, \mu_{i}\right)$. We do so in two steps: (1) we show that the optimal Pricing Mappings Mechanism can be represented by an element in a compact set; (2) we use compactness arguments to take the limit of such mechanism obtaining a mechanism for context $(u, \mu)$.

Compact representation: The revenue $R_{c}\left(u_{i}, \mu_{i}\right)$ is the solution of a variant the $\mathbf{L P}_{\mathbf{2}}$ described above. As the original $\mathbf{L P}_{\mathbf{2}}$, it has $O\left(|\Theta|^{2}\right)$ constraints and therefore has an optimal solution that has support $O\left(|\Theta|^{2}\right)$. Now, the optimal mechanism for context $\left(u_{i}, \mu_{i}\right)$ can be represented by a price vector $t^{i} \in \mathbb{R}_{+}^{\Omega}$ and random variables $Y_{\theta}^{i}$ with $O\left(|\Theta|^{2}\right)$ support. This means that each of those random variables can be represented as $|\Omega|$ distributions $\left(\psi_{\theta}^{\omega}\right)^{i} \in \Delta\left(\left[O\left(|\Theta|^{2}\right)\right]\right)$. So, the set of variables $\left\{Y_{\theta}^{i}\right\}_{\theta \in \Theta}$ admits a representation as an element of a compact set. Now, we need to argue that the set of vectors $t^{i}$ are bounded and therefore can be also represented as elements of a compact set.

As for the price vector $t^{i}$, notice that $t^{i} \leq \xi^{i}$ for any individually rational mechanism, where $\xi^{i}$ is the surplus vector for context $\left(u_{i}, \mu_{i}\right)$. If $\xi$ is the surplus vector for context $(u, \mu)$ then for $i \geq i_{0}$, then $\left\|\xi^{i}-\xi\right\|_{\infty} \leq 1$. For the $i \geq i_{0}$, therefore $t^{i}$ is in the compact $\prod_{\theta}[0, \xi(\theta)+1]$.

Limit mechanism: Now, for each context $\left(u_{i}, \mu_{i}\right)$, the optimal mechanism can be represented a point $\mathcal{M}_{i} \in K$ where $K$ is a compact set. Therefore, passing to a subsequence if necessary, we can assume that $\mathcal{M}_{i} \rightarrow \mathcal{M}$. Now, since IR and IC properties are preserved in the limit (since they are continuous properties), $\mathcal{M}$ is a valid Pricing Mappings Mechanism for context $(u, \mu)$ and 
its revenue is at least the limit of the revenue extracted by $\mathcal{M}_{\rangle}$on $\left(u_{i}, \mu_{i}\right)$. Therefore, $R_{c}(u, \mu) \geq$ $\lim \sup R_{c}\left(u_{i}, \mu_{i}\right)$.

An even simpler compactness argument can be formulated for $R_{e}$. For $R_{p}$ we need to add a mild non-degeneracy assumptions: we say that a context $(u, \mu)$ is non-degenerated if $\mu(\omega, \theta)>0, \forall \omega, \theta$.

Lemma D.3 The revenue function $R_{p}: \mathcal{C} \rightarrow \mathbb{R}$ is upper-semicontinuous in the neighborhood of all non-degenerated context.

Proof : As in the proof of Lemma D.2, the main goal is to show that the set of optimal mechanisms for contexts $\left(u_{i}, \mu_{i}\right)$ are members of a compact set and then proceed as in the second part of the proof of that lemma. For the first part, we proceed for the $Y_{\theta}^{i}$ as in Lemma D.2. But we need a different argument to show that the transfers in the optimal mechanism are bounded.

Given a non-degenerated context, let $\delta=\frac{1}{2} \min _{\omega, \theta} \mu(\theta \mid \omega)$, then if $\left(u_{i}, \mu_{i}\right) \rightarrow(u, \mu)$, then for $i \geq i_{0}$, then $\mu_{i}(\theta \mid \omega) \geq \delta$ for all $\omega, \theta$. Increasing $i_{0}$ if necessary, we can also assume that $\left\|\xi^{i}-\xi\right\|_{\infty} \leq 1$ for $i \geq i_{0}$. So, if $t_{\theta}^{i}(q)$ is the payment of buyer $\theta$ for posterior $q$ in the optimal mechanism for context $\left(u_{i}, \mu_{i}\right)$, then $\tilde{t}_{\theta}^{i}(q)=x_{\theta}^{i}(q) \cdot t_{\theta}^{i}(q)$ is in the compact set $\prod_{i}\left[0, \frac{1}{\delta}(\xi(\theta)+1)\right]$ for all individually rational mechanism, since:

$$
\sum_{q} v_{\theta}\left(D_{\theta} q\right) x_{\theta}(q)-\left(\mathbb{1}^{t} D_{\theta} q\right) \tilde{t}_{\theta}(q) \geq v_{\theta}\left(D_{\theta} p\right)
$$

implies that:

$$
\begin{aligned}
\xi(\theta)+1 & \geq \xi^{i}(\theta) \geq \frac{1}{\mu^{i}(\theta)}\left[\sum_{q} v_{\theta}^{i}\left(D_{\theta} q\right) x_{\theta}^{i}(q)-v_{\theta}^{i}\left(D_{\theta} p\right)\right] \\
& \geq \sum_{q}\left(\mathbb{1}^{t} D_{\theta} q\right) \cdot \tilde{t}_{\theta}^{i}(q) \geq\left(\mathbb{1}^{t} D_{\theta} q\right) \cdot \tilde{t}_{\theta}^{i}(q) \geq \delta \cdot \tilde{t}_{\theta}^{i}(q)
\end{aligned}
$$

Notice that inequalities crucially depend on $\tilde{t}_{\theta}^{i}(q)$ being non-negative.

The following theorem summarizes the previous discussion:

Theorem D.4 The revenue functions $R_{c}, R_{e}: \mathcal{C} \rightarrow \mathbb{R}$ are continuous and the revenue function $R_{p}: \mathcal{C} \rightarrow \mathbb{R}$ is continuous around any non-degenerated context.

\section{D.2 Approximating Revenue}

We know from Example B.1 that the Sealed Envelope Mechanism performs very poorly in terms of revenue when compared to the optimal mechanism, even if the $\omega, \theta$-signals are independent. A natural question is how do the other mechanisms perform in comparison to the optimal mechanism. The Pricing Mappings Mechanism is simple and allows for a much more natural implementation then the Pricing Outcomes. Unfortunately, as we show in this section, both the Pricing Mappings Mechanism and the Pricing Outcomes with no positive transfers Mechanism can perform very poorly compared to the Pricing Outcomes Mechanism for certain contexts:

Lemma D.5 The revenue extracted by a Pricing Mappings Mechanism and by a Pricing Outcomes with no positive transfers Mechanism is at least a $1 /|\Theta|$-fraction of the optimal mechanism:

$$
R_{c}(u, \mu) \geq \frac{1}{|\Theta|} R(u, \mu), \quad R_{p}(u, \mu) \geq \frac{1}{|\Theta|} R(u, \mu)
$$

and those bounds are tight up to constant factors. 
The bound is trivial and it follows directly from the fact that the optimal revenue is bounded by the surplus, and extracting full surplus from a single type is trivial. Now, we show that the bound is tight using the continuity of $R_{c}(\cdot)$ and $R_{p}(\cdot)$.

Example D.6 In this example we show that there exist a context $(u, \mu)$ such that $R_{c}(u, \mu) \leq$ $O(1 /|\Theta|) R(u, \mu)$ and $R_{p}(u, \mu) \leq O(1 /|\Theta|) R(u, \mu)$.

For $\Omega=\Theta=[n]$, we present a non-degenerated context $(u, \mu)$ where $(\omega, \theta)$ are independent and the optimal revenue is a $1 / n$-fraction of the full surplus $\sum_{\theta} \mu(\theta) \xi(\theta)$. For this context:

$$
R(u, \mu)=R_{p}(u, \mu)=R_{c}(u, \mu)=\frac{1}{n} \sum_{\theta} \mu(\theta) \xi(\theta)
$$

Now, if one sees $\mu$ as a $n \times n$-matrix and $\eta$ is any $n \times n$-matrix of full rank with $\sum_{\omega, \theta} \eta(\omega, \theta)=0$, then for any small $t>0, \mu+t \eta$ represents a probability distribution over $\Theta \times \Omega$ close to $\mu$ but with $\operatorname{rank}(\mu+t \eta)=n$, therefore, the optimal mechanism extracts full revenue (Theorem 4.2). In other words:

$$
R(u, \mu+t \eta)=\sum_{\theta} \mu(\theta) \xi_{t}(\theta)
$$

where $\xi_{t}$ is the surplus for context $(u, \mu+t \eta)$. By continuity:

$$
\lim _{t \rightarrow 0+} R_{c}(u, \mu+t \eta)=R_{c}(u, \mu)=\frac{1}{n} \sum_{\theta} \mu(\theta) \xi(\theta)
$$

while

$$
\lim _{t \rightarrow 0+} R(u, \mu+t \eta)=\sum_{\theta} \mu(\theta) \xi(\theta)
$$

showing that the bound it tight. So, all we need to so is to exhibit a context where the optimal revenue is a $\beta / n$ fraction of the full surplus for some constant $\beta$.

Let $\Omega=\Theta=A=[n], u(\theta, \omega, a)=v_{\theta} \cdot \mathbb{1}\{a=\omega\}, v_{\theta}=2^{\theta}, \mu(\omega)=\frac{1}{n}$ and $\mu(\theta)=\frac{2^{-\theta}}{1-2^{-n}}$. The surplus is given by $\xi(\theta)=2^{\theta}\left(1-\frac{1}{n}\right)$, so $\sum_{\theta} \mu(\theta) \xi(\theta)=\frac{n-1}{1-2^{-n}}$. The revenue optimal mechanism for this context is a Pricing Mappings Mechanism.

Using the construction in the Interesting Posteriors Lemma (Lemma 3.5) we can search for a solution where the only posteriors in the support are of the form $q_{X}(\omega)=\frac{1}{|X|}$ for $\omega \in X$ and $q_{X}(\omega)=0$ otherwise, where $X$ is any subset of $[n]$. Therefore we can solve the primal LP only considering the $x_{\theta}\left(q_{X}\right)$ variables, i.e., we need to solve:

$$
\begin{aligned}
& \max \sum_{\theta} \frac{2^{-\theta}}{1-2^{-n}} t_{\theta} \text { s.t. } \\
& -t_{\theta}+\sum_{X \subseteq[n]} x_{\theta}\left(q_{X}\right) \frac{v_{\theta}}{|X|} \geq \frac{v_{\theta}}{n}, \\
& -t_{\theta}+\sum_{X \subseteq[n]} x_{\theta}\left(q_{X}\right) \frac{v_{\theta}}{|X|} \geq-t_{\theta^{\prime}}+\sum_{X \subseteq[n]} x_{\theta^{\prime}}\left(q_{X}\right) \frac{v_{\theta}}{|X|}, \\
& \sum_{\theta} x_{\theta}\left(q_{X}\right)=1, x_{\theta}\left(q_{X}\right) \geq 0
\end{aligned}
$$

Notice one can define $\hat{x}_{\theta}=\sum_{X \subseteq[n]} \frac{1}{|X|} x_{\theta}\left(q_{X}\right)$ and re-write the LP as: 


$$
\begin{aligned}
& \max \sum_{\theta} \frac{2^{-\theta}}{1-2^{-n}} t_{\theta} \text { s.t. } \\
& \quad-t_{\theta}+\hat{x}_{\theta} v_{\theta} \geq v_{\theta} / n, \\
& \quad-t_{\theta}+\hat{x}_{\theta} v_{\theta} \geq-t_{\theta^{\prime}}+\hat{x}_{\theta^{\prime}} v_{\theta}, \\
& 1 / n \leq \hat{x}_{\theta} \leq 1
\end{aligned}
$$

Now it suffices to show that the solution of the above LP is bounded by a constant as $n$ grows. This can be seen very easily by showing a feasible dual. We have that:

$$
\left[-t_{1}+\hat{x}_{1} v_{1}-\frac{v_{1}}{n}\right]+\sum_{\theta=1}^{n-1} 2^{-\theta}\left[-t_{\theta+1}+\hat{x}_{\theta+1} v_{\theta+1}+t_{\theta}-\hat{x}_{\theta} v_{\theta+1}\right] \geq 0
$$

substituting $v_{\theta}=2^{\theta}$ and simplifying the expression, we get:

$$
\sum 2^{-\theta} t_{\theta} \leq 2 \hat{x}_{n}-\frac{t_{n}}{2^{n}}-\frac{2}{n} \leq 2
$$

One might also wonder what is the relation between $R_{c}$ and $R_{p}$. Clearly $R_{c}(u, \mu) \geq \frac{1}{|\Theta|} R_{p}(u, \mu)$. The following simple example shows that this bound is tight.

Example D.7 In this example we show that there exists a context $(u, \mu)$ such that $R_{c}(u, \mu) \leq$ $O(1 /|\Theta|) R_{p}(u, \mu)$.

We consider in this example the case where the seller learns exactly the type of the buyer. We encode this information in his signal $\omega$. It will have two components: $\omega_{1}$ which is the information the buyer is interested and $\omega_{2}$ which tells the seller the type $\theta$ of the buyer. A Pricing Outcomes Mechanism will be able to explore this information in order to extract full surplus from the buyer, but a pricing contracts cannot.

Let $\Omega_{0}=\{0,1\}$ and $\Theta_{0}=[n]$ and consider a probability distribution $\mu_{0}$ on $\Omega_{0} \times \Theta_{0}$ where $\mu_{0}\left(\omega_{0}, \theta_{0}\right)=\frac{1}{2^{1-\theta_{0}}} \cdot \frac{1}{1-2^{-n}}$. Now, define utilities of the buyer as functions of $u_{0}\left(\omega_{0}, \theta_{0}, a\right)$. As usual, we define as a piecewise-linear function $v_{0, \theta_{0}}:[0,1] \rightarrow \mathbb{R}$. Consider the function interpolating $\left(0,2^{\theta_{0}}\right),\left(\frac{1}{2}, 0\right),\left(1,2^{\theta_{0}}\right)$. Now, let's define $\Theta=\Theta_{0}$ and $\Omega=\Omega_{0} \times \Theta_{0}$, and if $\omega=\left(\omega_{1}, \omega_{2}\right)$, then we define a probability distribution $\mu$ on $\Omega \times \Theta$ as $\mu(\omega, \theta)=\mu_{0}\left(\omega_{1}, \theta\right)$ and $u(\omega, \theta, a)=u_{0}\left(\omega_{1}, \theta, a\right)$.

By using a Pricing Mappings Mechanism, the seller is not able to use the information in $\omega_{2}$, and therefore he can only extract $O(1)$ revenue. If the seller is able to price signals, he can design the contract $\theta$ such that the price of $(0, \theta)$ and $(1, \theta)$ is $2^{\theta}-\epsilon$ and the price of the signals $\left(\omega_{0}, \theta^{\prime}\right)$ is $\infty$ for $\theta^{\prime} \neq \theta$. Therefore, buyers will be forced to buy the contract for their correct type.

The implementation above casts the mechanism in the general "Pricing Outcomes" form given by Theorem 4.1, but a more natural implementation would be for the seller to learn the $\theta=\omega_{2}$ and then announce price $2^{\theta}-\epsilon$ for the full information signal.

\section{E Proofs omitted from Section 5}

Claim E.1 In the interactive protocol presented in Example 5.3 the optimal strategy for an uncommitted buyer is to play left ( $t o$ node $t_{1}$ ) whenever $\theta=0$ and play right (to node $s_{2}$ ) whenever $\theta=1$, and then follow the protocol (make the transfers when asked) without defecting.

Proof : It is simple to see that a buyer of type $\theta=1$ will not play left at the root, since his surplus for the information is only $\xi(0)=0.4<0.533$. A buyer of type $\theta=0$ playing left and not 
defecting gets the full information and pays 0.533 , so his utility is $1-0.533=0.467$. information and pays 0.533 , so his utility is $1-0.533=0.467$. To complete the proof we still need to show that a buyer of type $\theta=0$ would not prefer to play right and that the buyer of type $\theta=1$ will follow the entire protocol.

In the node $s_{2}$, upon receiving $\ell_{5}$ both buyer types update their beliefs to $\omega=1$. Now, upon receiving $t_{2}$, they update their beliefs about $\omega$ to: $\mathbb{P}\left(\omega \mid \theta, t_{2}\right)=\mu(\omega \mid \theta) \cdot \mathbb{P}\left(t_{2} \mid \omega\right) / \sum_{\omega^{\prime}}\left(\mu\left(\omega^{\prime} \mid \theta\right) \cdot \mathbb{P}\left(t_{2} \mid \omega^{\prime}\right)\right)$. Doing the calculations, we get that $\mathbb{P}\left(\omega=1 \mid \theta=0, t_{2}\right)=0.1$ and $\mathbb{P}\left(\omega=1 \mid \theta=1, t_{2}\right)=0.2$. By the shape of the $v_{\theta}(\cdot)$ function, no player has increase in utility by this signal. Hence, a buyer of type $\theta=0$ would not play right and then defect in $t_{2}$. At this point (node $t_{2}$ ) the surplus for knowing the value of $\omega$ exactly is 0.9 for $\theta=0$ and 0.8 for $\theta=1$. So, it is clear that type $\theta=1$ would pay the amount of 0.8 to get the signal revealed.

Now, we are left with the question of whether the player of type $\theta=0$ would not prefer to play right. In both cases (left and right), he gets the full information about the value of $\omega$. Now, we need to compare which one has the smallest expected price in his perspective. The price of the left path is 0.533 and the left path has price $0.8 \cdot \mathbb{P}\left(t_{2} \mid \theta=0\right)=\frac{2}{3} \cdot 0.8=0.5333 \ldots$

\section{E.1 Proof of Theorem 5.4}

To prove Theorem 5.4 we consider the setting of Example 5.3 .

We first observe that it is possible to extract revenue 0.5 from committed buyers using a Pricing Outcomes with No Positive Transfers Mechanism. The interim belief of buyer of type 0 signal is $\mu(\omega=1 \mid \theta=0)=0.4$, so his surplus for the information is $\xi(0)=0.6$, while for buyer of type 1 his interim belief is 0.6 and his surplus is $\xi(1)=0.4$. By Theorem 4.2 we can extract the full surplus $\frac{1}{2} \xi(0)+\frac{1}{2} \xi(1)=0.5$ from committed buyers. This can be done by a Pricing Outcomes with No Positive Transfers Mechanism in which the seller reveals the value of $\omega$ and charges $t(\omega)$, where $t(0)=1$ and $t(1)=0$.

The next lemma completes the proof of the theorem.

Lemma E.2 For the context $(u, \mu)$ with correlated $\omega$ and $\theta$ that is defined in Example 5.3 it hold that there exists some $\delta>0$ such that the revenue of any protocol for uncommitted buyers that has no positive transfers is at most $0.5-\delta$.

Proof : Fix $\delta=10^{-10} \ll 10^{-2}=\epsilon$. Let's assume that there is a protocol with no positive transfers that is able to extract revenue $0.5-\delta$ from uncommitted buyers. We can assume by Theorem 2.6 that the protocol has the following format: the root is a buyer node with two children and all the other nodes of the tree are either seller or transfer nodes. We can also assume that the optimal strategy for the $\theta=0$ buyer is to take the left path and follow the protocol until then end and that the optimal strategy for the $\theta=1$ buyer is to take the right path and follow the protocol until the end.

Now, since the surplus of the types are 0.6 and 0.4 respectively, the protocol should extract at least $0.6-2 \delta$ from buyer of type 0 and at least $0.4-2 \delta$ from buyer of type 1 . We want to claim that in such a setting, a buyer of type $\theta=0$ can benefit from taking the right path (i.e., behaving like a buyer of type $\theta=1$ ) paying strictly less then $0.6-2 \delta$. This generates a contradiction.

Now, we start analyzing the change in utility for $\theta=0$ if he does as $\theta=1$, i.e. takes the right path. Let $L$ be the set of leaves that are in the right side of the root. And let $\psi^{\omega} \in \Delta(L)$ for be the distributions induced on the leaves for each $\omega \in\{0,1\}$. Now, if the output of the protocol is 
leaf $\ell$ the buyer of type $\theta$ updates his belief to:

$$
\mathbb{P}(\omega \mid \ell, \theta)=\frac{\mu(\omega \mid \theta) \psi^{\omega}(\ell)}{\sum_{\omega^{\prime}} \mu\left(\omega^{\prime} \mid \theta\right) \psi^{\omega^{\prime}}(\ell)}
$$

In vector notation, the posterior of a buyer of type $\theta$ upon observing a leaf $\ell$ of the right path is:

$$
q^{\theta, \ell}=\frac{A^{\ell} q^{\theta}}{\mathbb{1}^{t} A^{\ell} q^{\theta}}, \text { where } A^{\ell}=\left[\begin{array}{ll}
\psi^{0}(\ell) & \\
& \psi^{1}(\ell)
\end{array}\right] \text { and } q^{\theta}=\left[\begin{array}{l}
\mu(\omega=0 \mid \theta) \\
\mu(\omega=1 \mid \theta)
\end{array}\right]
$$

He obtains this posterior with probability $\sum_{\omega^{\prime}} \mu\left(\omega^{\prime} \mid \theta\right) \psi^{\omega^{\prime}}(\ell)=\mathbb{1}^{t} A^{\ell} q^{\theta}$. Therefore, his total value (not counting payments) for taking the right path is: $\sum_{\ell}\left(\mathbb{1}^{t} A^{\ell} q^{\theta}\right) \cdot v\left(\frac{A^{\ell} q^{\theta}}{\mathbb{1}^{t} A^{\ell} q^{\theta}}\right)$. The previous expression has a slightly notation abuse: we defined $v:[0,1] \rightarrow \mathbb{R}_{+}$. We overload the function $v$ with $v: \Delta(\Omega) \rightarrow \mathbb{R}_{+}$such that for $\left[\begin{array}{c}q(0) \\ q(1)\end{array}\right]$ we define $v(q)=v(q(1))$.

Fact E.3 If $\sum_{\ell}\left(\mathbb{1}^{t} A^{\ell} q^{1}\right) \cdot v\left(q^{1, \ell}\right) \geq 1-2 \delta$, then it must be the case that $\sum_{\ell}\left(\mathbb{1}^{t} A^{\ell} q^{0}\right) \cdot v\left(q^{0, \ell}\right) \geq 1-36 \cdot \delta$. In other words, if a buyer of type $\theta=1$ can extract value very close to 1 from learning the leaves reached by the protocol, then a buyer of type $\theta=0$ should also be able to extract values very close to 1 from it.

Proof : First let $L_{1}=\left\{\ell ; \sqrt{2 \delta}<q^{1, \ell}(1)<1-\sqrt{2 \delta}\right\}$. First, we claim that $\sum_{\ell \in L_{1}} \mathbb{1}^{t} A^{\ell} q^{1}<\sqrt{2 \delta}$. If not, then:

$$
\begin{aligned}
1-2 \delta & <\sum_{\ell}\left(\mathbb{1}^{t} A^{\ell} q^{1}\right) \cdot v\left(q^{1, \ell}\right) \leq \sum_{\ell \in L_{1}}\left(\mathbb{1}^{t} A^{\ell} q^{1}\right) \cdot v\left(q^{1, \ell}\right)+\sum_{\ell \notin L_{1}}\left(\mathbb{1}^{t} A^{\ell} q^{1}\right) \cdot v\left(q^{1, \ell}\right) \leq \\
& \leq(1-\sqrt{2 \delta}) \sum_{\ell \in L_{1}}\left(\mathbb{1}^{t} A^{\ell} q^{1}\right)+1 \cdot \sum_{\ell \notin L_{1}}\left(\mathbb{1}^{t} A^{\ell} q^{1}\right)=1-\sqrt{2 \delta} \sum_{\ell \in L_{1}}\left(\mathbb{1}^{t} A^{\ell} q^{1}\right) \leq \\
& \leq 1-\sqrt{2 \delta} \cdot \sqrt{2 \delta}=1-2 \delta
\end{aligned}
$$

using the fact that $v(q) \leq \max \{q, 1-q\}$ and the fact that $\sum_{\ell} A^{\ell}=\left[{ }^{1}{ }_{1}\right]$ by the definition of $A^{\ell}$. Now, in order to get an upper bound for $\sum_{\ell}\left(\mathbb{1}^{t} A^{\ell} q^{0}\right) \cdot v\left(q^{0, \ell}\right)$ we focus on $\ell \notin L_{1}$. For those vectors, we still need to bound $v\left(q^{0, \ell}\right)$. We consider two cases.

- case 1: $q^{1, \ell}(1) \leq \delta^{\prime}:=\sqrt{2 \delta}$.

$$
\delta^{\prime} \geq q^{1, \ell}(1)=\frac{A_{11}^{\ell} q^{1}(1)}{A_{00}^{\ell} q^{1}(0)+A_{11}^{\ell} q^{1}(1)}=\frac{A_{11}^{\ell} 0.6}{A_{00}^{\ell} 0.4+A_{11}^{\ell} 0.6} \Rightarrow \frac{A_{00}^{\ell}}{A_{11}^{\ell}} \geq \frac{3\left(1-\delta^{\prime}\right)}{2 \delta^{\prime}} \geq \frac{1}{2 \delta^{\prime}}
$$

Now, calculating $q^{0, \ell}(1)$, we get:

$$
q^{0, \ell}(1)=\frac{A_{11}^{\ell} q^{0}(1)}{A_{00}^{\ell} q^{0}(0)+A_{11}^{\ell} q^{0}(1)}=\frac{A_{11}^{\ell} 0.4}{A_{00}^{\ell} 0.6+A_{11}^{\ell} 0.4} \leq \frac{0.4}{\left(1 / 2 \delta^{\prime}\right) 0.6+0.4} \leq 2 \delta^{\prime}
$$

Therefore: $v\left(q^{0, \ell}\right) \geq 1-9\left(2 \delta^{\prime}\right)=1-18 \cdot \delta^{\prime}$

- case 2: $q^{1, \ell}(1) \geq 1-\delta^{\prime}=1-\sqrt{2 \delta}$.

$$
\delta^{\prime} \geq q^{1, \ell}(0)=\frac{A_{00}^{\ell} q^{1}(0)}{A_{00}^{\ell} q^{1}(0)+A_{11}^{\ell} q^{1}(1)}=\frac{A_{00}^{\ell} 0.4}{A_{00}^{\ell} 0.4+A_{11}^{\ell} 0.6} \Rightarrow \frac{A_{11}^{\ell}}{A_{00}^{\ell}} \geq \frac{2\left(1-\delta^{\prime}\right)}{3 \delta^{\prime}} \geq \frac{1}{3 \delta^{\prime}}
$$


Now, calculating $q^{0, \ell}(0)$, we get:

$$
q^{0, \ell}(0)=\frac{A_{00}^{\ell} q^{0}(0)}{A_{00}^{\ell} q^{0}(0)+A_{11}^{\ell} q^{0}(1)}=\frac{A_{00}^{\ell} 0.6}{A_{00}^{\ell} 0.6+A_{11}^{\ell} 0.4} \leq \frac{0.6}{0.6+0.4\left(1 / 3 \delta^{\prime}\right)} \leq 5 \delta^{\prime}
$$

Therefore: $v\left(q^{0, \ell}\right) \geq 1-\left(5 \delta^{\prime}\right)$

The important fact is that $v\left(q^{0, \ell}\right) \geq 1-18 \sqrt{2 \delta}$ for each $\ell \notin L_{1}$. Now, we are ready to bound the value that type $\theta=0$ gets from the right path:

$$
\sum_{\ell \notin L_{1}}\left(\mathbb{1}^{t} A^{\ell} q^{0}\right) \cdot v\left(q^{0, \ell}\right) \geq \sum_{\ell \in L_{1}}\left(\mathbb{1}^{t} A^{\ell} q^{0}\right) \cdot v\left(q^{0, \ell}\right) \geq(1-\sqrt{2 \delta}) \cdot(1-18 \sqrt{2 \delta}) \geq 1-36 \cdot \delta
$$

Now, for the next step, we bound the total expected payment of buyer $\theta=0$ if he decides to deviate to the right path and follow the protocol until the end. In order to do so, let $N$ the set of transfer nodes in the left path and for each $i \in N$, let $t_{i}$ be the amount associated with it. Also, let $\mathbb{P}(i \mid \omega)$ be the probability that a buyer following the right branch of the tree reaches $i$ condioned on $\omega$. Define also the matrix:

$$
A^{i}=\left[\begin{array}{cc}
\mathbb{P}(i \mid \omega=0) & 0 \\
0 & \mathbb{P}(i \mid \omega=1)
\end{array}\right]
$$

Now, remember we had fixed in the beginning a constant $\epsilon \gg \delta$ but still $\epsilon \ll 1$. Now the expected payment of player of type $\theta$ in the right branch of the protocol is given by:

$$
\sum_{\omega} \mu(\omega \mid \theta) \sum_{i} \mathbb{P}(i \mid \omega) t_{i}=\sum_{i}\left(\mathbb{1}^{t} A^{i} q^{\theta}\right) \cdot t_{i}
$$

We know that player of type $\theta=1$ pays at least $0.4-2 \delta$, so $\sum_{i}\left(\mathbb{1}^{t} A^{i} q^{1}\right) \cdot t_{i} \geq 0.4-2 \delta$. Now, we show that the payment of buyer $\theta=0$ if he switches to the right branch of the protocol (i.e. moves as a buyer of type $\theta=1$ ) is $1-O(\epsilon)$, giving him utility $O(\epsilon) \gg 2 \delta$ for doing so. We formalize this below.

Fact E.4 If the payment of player of type $\theta=1$ is $\sum_{i}\left(\mathbb{1}^{t} A^{i} q^{1}\right) \cdot t_{i} \leq 0.4$, then the expected payment $\sum_{i}\left(\mathbb{1}^{t} A^{i} q^{0}\right) \cdot t_{i}$ of the player of type $\theta=0$ after moving is at most $0.6-\epsilon(0.4-\epsilon)$.

Proof : We will decompose the sum $\sum_{i}\left(\mathbb{1}^{t} A^{i} q^{0}\right) \cdot t_{i}$ in two parts and bound each of them separately. First, we divide the transfer nodes in two classes:

$$
\begin{aligned}
& N_{1}=\left\{i \in N ; \mathbb{1}^{t} A^{i} q^{0} \leq\left(\frac{3}{2}-\epsilon\right) \mathbb{1}^{t} A^{i} q^{1}\right\} \\
& N_{2}=\left\{i \in N ; \mathbb{1}^{t} A^{i} q^{0}>\left(\frac{3}{2}-\epsilon\right) \mathbb{1}^{t} A^{i} q^{1}\right\}
\end{aligned}
$$

First note that for all $i$ (even $i \in N_{2}$ ), $\mathbb{1}^{t} A^{i} q^{0} \leq \frac{3}{2} \mathbb{1}^{t} A_{i} q^{1}$ simply because $q^{0} \leq \frac{3}{2} q^{1}$ componentwise. Now, we can write:

$$
\sum_{i}\left(\mathbb{1}^{t} A^{i} q^{0}\right) \cdot t_{i} \leq \sum_{i \in N_{1}}\left(\frac{3}{2}-\epsilon\right) \cdot \mathbb{1}^{t} A^{i} q^{1}+\sum_{i \in N_{2}} \frac{3}{2} \cdot \mathbb{1}^{t} A^{i} q^{1}
$$

If we show that most of $\sum_{i}\left(\mathbb{1}^{t} A^{i} q^{1}\right) \cdot t_{i}$ is concentrated on $N_{1}$ we are done.

Now, consider some transfer node $i \in N_{2}$ and recall that $q^{0}=\left[\begin{array}{l}0.6 \\ 0.4\end{array}\right]$ and $q^{1}=\left[\begin{array}{l}0.4 \\ 0.6\end{array}\right]$. Therefore:

$$
i \in N_{2} \Leftrightarrow 0.6 A_{00}^{i}+0.4 A_{11}^{i} \geq\left(\frac{3}{2}-\epsilon\right)\left(0.4 A_{00}^{i}+0.6 A_{11}^{i}\right) \Leftrightarrow \frac{A_{11}^{i}}{A_{00}^{i}} \leq \frac{4 \epsilon}{5-6 \epsilon}
$$


Observe also that once in a node $i \in N_{2}$, a buyer of type $\theta=1$ is willing to follow the protocol until the end, the expected amount he is paying by following this node, must be at least as big as his surplus for the full information at that node. Formally:

$$
1-v\left(\frac{A^{i} q^{1}}{\mathbb{1}^{t} A^{i} q^{1}}\right) \geq t_{i}+\sum_{j \in N(i)} t_{j} \cdot \frac{\mathbb{1}^{t} A^{j} q^{1}}{\mathbb{1}^{t} A^{i} q^{1}}
$$

where $N(i)$ is the set of transfer nodes that have $i$ as an ancestor (excluding $i$ itself). In order to justify the term $\frac{\mathbb{1}^{t} A^{j} q^{1}}{\mathbb{1}^{t} A^{i} q^{1}}$, notice that if $i$ is an ancestor of $i$ then the probability of reaching $j$ given $i$ is given by:

$$
\mathbb{P}(j \mid i, \theta)=\frac{\mathbb{P}(i, j \mid \theta)}{\mathbb{P}(i \mid \theta)}=\frac{\mathbb{P}(j \mid \theta)}{\mathbb{P}(i \mid \theta)}=\frac{\mathbb{1}^{t} A^{j} q^{1}}{\mathbb{1}^{t} A^{i} q^{1}}
$$

Now, given $i \in N_{2}$, since the matrix $A^{i}$ is of the form [ ${ }^{O(1)}{ }_{O(\epsilon)}$ ], the posterior probability of each player in that node is $O(\epsilon)$, so $v(q)=w^{t} q$ where $w=\left[\begin{array}{c}1 \\ -8\end{array}\right]$. Therefore, for $i \in N_{2}$, we can re-write equation (3) as:

$$
t_{i} \cdot\left(\mathbb{1}^{t} A^{i} q^{1}\right)+\sum_{j \in N(i)} t_{j} \cdot\left(\mathbb{1}^{t} A^{j} q^{1}\right) \leq \hat{w}^{t} A^{i} q^{1}
$$

where $\hat{w}=\mathbb{1}-w=\left[\begin{array}{l}0 \\ 9\end{array}\right]$. Now, let $N_{2}^{g}$ be the nodes in $N_{2}$ that have no ancestor in $N_{2}$. Therefore:

$$
\sum_{i \in N_{2}} t_{i} \cdot\left(\mathbb{1}^{t} A^{i} q^{1}\right) \leq \sum_{i \in N_{2}^{g}}\left[t_{i} \cdot\left(\mathbb{1}^{t} A^{i} q^{1}\right)+\sum_{j \in N(i)} t_{j} \cdot\left(\mathbb{1}^{t} A^{j} q^{1}\right)\right] \leq \sum_{i \in N_{2}^{g}} \hat{w}^{t} A^{i} q^{1} \leq 9 \cdot \sum_{i \in N_{2}^{g}} A_{11}^{i} \cdot 0.6
$$

Now, we know by equation (2) that:

$$
\sum_{i \in N_{2}^{g}} A_{11}^{i} \leq \sum_{i \in N_{2}^{g}} \frac{4 \epsilon}{5-6 \epsilon} \cdot A_{00}^{i} \leq \epsilon \sum_{i \in N_{2}^{g}} \mathbb{P}(i \mid \omega=0) \leq \epsilon
$$

where we are using the definition that $A_{00}^{i}$ is the probability that the protocol reached node $i$ given $\omega=0$ and we are using the fact that $\sum_{i \in N_{2}^{g}} \mathbb{P}(i \mid \omega=0) \leq 1$ since the protocol reaching $i \in N_{2}^{g}$ are disjoint events, since for all paths in the protocol-tree there is at most one node in $N_{2}^{g}$. Therefore: $\sum_{i \in N_{2}} t_{i} \cdot\left(\mathbb{1}^{t} A^{i} q^{1}\right) \leq 9 \epsilon$, which allows us to finish the bound in equation (11):

$$
\sum_{i}\left(\mathbb{1}^{t} A^{i} q^{0}\right) \cdot t_{i} \leq \sum_{i \in N_{1} \cup N_{2}}\left(\frac{3}{2}-\epsilon\right) \cdot \mathbb{1}^{t} A^{i} q^{1}+\epsilon \sum_{i \in N_{2}} \cdot \mathbb{1}^{t} A^{i} q^{1} \leq 0.4\left(\frac{3}{2}-\epsilon\right)-\epsilon^{2}=0.6-\epsilon(0.4-\epsilon)
$$

Taking Fact E.3 and Fact E.4 together, it is clear that there cannot be a protocol extracting $0.5-\delta$ from uncommitted buyers without transfers from the seller to the buyer. Summarizing the argument, if there were such protocol, then the buyer of type $\theta=1$ would be faced with the option of getting almost full information (i.e. getting a signal that would give him at least $0.4-2 \delta$ value) and would pay at most 0.4 . If a buyer of type $\theta=0$ pretends to be of type $\theta=1$, he guarantees by fact Fact E.3 a contract that has almost full information (gives him value at least $1-36 \delta$ ) and charges him at most $0.6-0.4 \epsilon+\epsilon^{2}$ (Fact E.4). This contradicts the fact that we can extract at least $0.6-2 \delta$ revenue from type $\theta=0$. 